\title{
High-order Stochastic Simulation of Complex Spatially Distributed Natural Phenomena
}

\author{
Hussein Mustapha · Roussos Dimitrakopoulos
}

Received: 22 June 2009 / Accepted: 27 May 2010 / Published online: 18 June 2010

(C) The Author(s) 2010. This article is published with open access at Springerlink.com

\begin{abstract}
Spatially distributed and varying natural phenomena encountered in geoscience and engineering problem solving are typically incompatible with Gaussian models, exhibiting nonlinear spatial patterns and complex, multiple-point connectivity of extreme values. Stochastic simulation of such phenomena is historically founded on second-order spatial statistical approaches, which are limited in their capacity to model complex spatial uncertainty. The newer multiple-point (MP) simulation framework addresses past limits by establishing the concept of a training image, and, arguably, has its own drawbacks. An alternative to current MP approaches is founded upon new high-order measures of spatial complexity, termed "high-order spatial cumulants." These are combinations of moments of statistical parameters that characterize non-Gaussian random fields and can describe complex spatial information. Stochastic simulation of complex spatial processes is developed based on high-order spatial cumulants in the high-dimensional space of Legendre polynomials. Starting with discrete Legendre polynomials, a set of discrete orthogonal cumulants is introduced as a tool to characterize spatial shapes. Weighted orthonormal Legendre polynomials define the so-called Legendre cumulants that are high-order conditional spatial cumulants inferred from training images and are combined with available sparse data sets. Advantages of the high-order sequential simulation approach developed herein include the absence of any distribution-related assumptions and pre- or post-processing steps. The method is shown to generate realizations of complex spatial patterns, reproduce bimodal data distributions, data variograms, and high-order spatial cumulants of the data. In addition, it is shown that the available hard data dominate the simulation process and have a definitive effect on the simulated realizations, whereas the training images are only used to fill in high-order relations that cannot be inferred from data. Compared to the MP framework, the pro-
\end{abstract}

H. Mustapha $(\bowtie) \cdot$ R. Dimitrakopoulos COSMO - Stochastic Mine Planning Laboratory, Department of Mining and Materials Engineering, McGill University, 3450 University St., Montreal, QC, Canada H3A 2A7

e-mail: hussein.mustapha@mcgill.ca 
posed approach is data-driven and consistently reconstructs the lower-order spatial complexity in the data used, in addition to high order.

Keywords Conditional sequential simulation · High-order spatial cumulants · Legendre polynomials

\section{Introduction}

Spatial uncertainty in the attributes of geological and other natural phenomena is frequently modeled using stochastic simulation of stationary and ergodic random fields, conditional to available data. Today's trends, developments, and applications in this area of problem solving focus on the so-called multiple-point simulation algorithms, such as the snesim (Strebelle 2002), filtersim (Zhang et al. 2006; Wu et al. 2008), and simpat (Arpat and Caers 2007) algorithms, and related extensions (Boucher 2009; Chugunova and Hu 2008; Mirowski et al. 2008; Remy et al. 2009; and others). Additional related new developments include Markov random field-based multiple-point type approaches (Daly 2004; Tjelmeland and Eidsvik 2004), kernel approaches (Scheidt and Caers 2009), and multiscale simulations based on discrete wavelet decomposition (Gloaguen and Dimitrakopoulos 2009; Chatterjee et al. 2009).

The development of multiple-point (or multipoint) models stems from the pragmatic need to tackle the effective modeling of complex nonlinear spatial patterns, deal with the noncompliance of natural phenomena with Gaussian models and, in general, distributional assumptions, and/or replicate the multiple-point connectivity of extreme values present in geological formations. These are all recognized important issues with substantial practical ramifications in most fields of application in geosciences and engineering. Conventional simulation methods (Goovaerts 1998; Chilès and Delfiner 1999; Remy et al. 2009; and others) are limited in their effectiveness of dealing with spatial complexity, largely because they are also limited to the two-point or second-order spatial statistical moments of the corresponding random field models employed. This limit has been recognized and documented since the early 1990s (Guardiano and Srivastava 1993; Tjelmeland 1998; Journel 1997). By spatial complexity, one may refer to, for example, curvilinear features in geologic domains controlling physical properties of rocks (such as ore concentrations) or overprints of successive geological events (such as high-grade ore in complex vein geometries), among others. Interestingly, efforts in the 1980s in dealing with geological complexity focused on the multiple-point covariance, or generalized covariance of linear combinations of locations in the context of nonstationary random fields with stationary increments of order $k$ and their simulation (Dimitrakopoulos 1990). However, the lack of the concept of a "training image" and the mathematical complexity of the related simulation methods limited the application of these developments.

The multiple-point methods noted above substantially address many of the limits of conventional methods; see, for example, the simulation of complex patterns in Boucher (2009). The ability to deal with complexity by MP methods is related to the 
introduction of the so-termed training images (TI) or analogues of the phenomenon under study; these serve as the source of underlying patterns, and a simulated realization should reproduce their probability of occurrence. Arguably, the conventional random field model is, in a sense, replaced by one where the TI is one of its parameters (Remy et al. 2009). Despite the advances in spatial modeling and simulation offered by the existing multiple-point approaches, there are interrelated drawbacks, which may be outlined as follows: (i) multiple-point algorithms are, arguably, somewhat ad hoc and lack a general mathematical framework; thus, theoretical questions of consistency may be raised; (ii) the choice of the training image is subjective and may be intrinsically unrealistic, while it overwrites the underlying spatial relations in data available. As a result, in the presence of a reasonable amount of hard data, conflicts between simulated realizations and the statistics of the data can become apparent; and (iii) to utilize information from a training image, MP algorithms use templates as a basis for modeling underlying spatial relations and patterns, that is, the spatial high-order moments of some corresponding non-Gaussian random field. The choice of templates may be arbitrary while templates appear geologically uninterpretable, unlike second-order spatial moments (David 1988). In summary, a welldefined spatial stochastic modeling framework dealing with the complex high-order geostatistical (spatial) description of complex and/or nonlinear geological and other natural phenomena is an avenue well worth exploring. A consistent mathematical model should ensure that the modeling process is data-driven, not training imagedriven, and thus avoids potential conflict between the spatial statistics of the data and simulated realizations.

Recently, Dimitrakopoulos et al. (2010) suggested the concept of high-order spatial cumulants and its use to characterize complex geological patterns. High-order cumulants are combinations of moment statistical parameters that characterize nonGaussian random variables (Billinger and Rosenblatt 1966) and may be seen as an extension of the well-known covariance function. The systematic definitions of spatial cumulants, including random variables, their moments and cumulants, nonGaussian spatial random fields, and their high-order spatial statistics are given in Dimitrakopoulos et al. (2010) along with duality relations between characteristics of cumulants as mathematical entities and the in situ spatial behavior of geological characteristics and patterns. The algorithm to efficiently calculate high-order spatial cumulants and additional examples can be found in Mustapha and Dimitrakopoulos (2010). In general, spatial cumulants of orders three to five are shown to be able to capture directional multiple-point periodicity, multiple-point connectivity (including connectivity of extreme values), and geometric characteristics and spatial architecture of two- and three-dimensional images and datasets. Most important in the abovementioned work are the specific relations shown between the order of the spatial cumulants and the lower-order moments that can make a simulation process consistent over a series of orders, that is, spatial cumulants are not some experimentally selected moments. This consistency does not appear to be the case with the existing MP methods. In addition, the above work shows that there is a wealth of spatial information quantified by high-order cumulants, which can therefore be carried through to the next step: a new high-order stochastic simulation framework such as the one presented herein. 
It is appropriate to note that the use of high-order cumulants are critical contributors to non-Gaussian and nonlinear modeling, where related developments include cumulants for signal filtering and deconvolution (Nikias and Petropulu 1993; Sadler et al. 1995; Delopoulos and Giannakis 1996; Zhang 2005), the estimation of the gravitational evolution of the cosmic distribution function (Gaztanaga et al. 2000), and conditional high-order cumulants in the high-precision astronomy (Bernardeau et al. 2002). A key justification for the use of cumulants in these fields and others is the wealth of information they contain compared to second-order statistical measures (Pan and Szapudi 2005), and their ability to support non-Gaussianity and nonlinearity in the context of modeling.

This paper contributes to the definition of the predictive aspects of non-Gaussian random field models, and, more specifically, a new simulation framework for complex nonlinear geological and other spatial patterns. This is based on the use of spatial cumulants in the high-dimensional space of Legendre polynomials (e.g., Lebedev 1965; Hosny 2007) and utilizes training images for information additional to the data without utilizing data transformations. In the following sections, a sequential simulation method using high-order spatial conditional cumulants is first introduced. Subsequently, the proposed general algorithm is presented. Finally, several numerical examples are presented to demonstrate the practical aspects and main features of the high-order simulation framework proposed. Discussion and conclusions follow.

\section{Sequential Simulation with High-order Spatial Cumulants}

Consider a stationary and ergodic random field $Z\left(x_{i}\right)$ or $Z_{i}, x_{i} \in \Omega \subseteq \mathrm{R}^{r}(r=1,2$, or 3) for $i=0, \ldots, N$, where $N$ is the number of points in a discrete grid $\left(D_{N}\right)$ and a set of conditioning data $d_{n}=\left\{Z\left(x_{\alpha}\right), \alpha=1, \ldots, n\right\}$. In addition, we introduce the sets $\Lambda_{i}$ such that $\Lambda_{0}=\left\{d_{n}\right\}, \Lambda_{i}=\Lambda_{i-1} \cup\left\{Z\left(x_{i-1}\right)\right\}$. Let $f_{\mathbf{Z}}\left(z_{0}, z_{1}, \ldots, z_{N}\right)$ be a probability distribution function associated with a multivariate process $\mathbf{Z}=$ $\left\{Z_{0}, Z_{1}, \ldots, Z_{N}\right\}$ at $\mathbf{x}=\left\{x_{0}, x_{1}, \ldots, x_{N}\right\}$. The multivariate distribution $f_{\mathbf{Z}}$ can be decomposed, based on the Bayes relation, into the product of univariate conditional distributions functions (Ripley 1987; Rosenblatt 1985; Rubinstein 1981; Law and Kelton 1999)

$$
\begin{aligned}
f_{\mathbf{Z}}\left(\mathbf{z} / \Lambda_{0}\right) & =f_{\mathbf{Z}}\left(z_{0}, z_{1}, z_{2}, \ldots, z_{N} / \Lambda_{0}\right) \\
& =f_{Z_{0}}\left(z_{0} / \Lambda_{0}\right) f_{Z_{1}}\left(z_{1} / \Lambda_{1}\right) \cdots f_{Z_{N}}\left(z_{N} / \Lambda_{N}\right),
\end{aligned}
$$

where $f_{Z_{i}}\left(z_{i} / \Lambda_{i}\right)$ is the conditional distribution of $Z_{i}$, given $\Lambda_{i}$. To generate a realization $\mathbf{z}=\left(z_{0}, \ldots, z_{N}\right)$ of $\mathbf{Z}$, a value $z_{0}$ is drawn for $Z_{0}$ based on $f_{Z_{0}}\left(z_{0} / \Lambda_{0}\right)$; then, $z_{i}, i=1, \ldots, N$, is drawn from the conditional probability function $f_{Z_{i}}\left(z_{i} / \Lambda_{i}\right)$. For additional details, see Journel (1994) and Dimitrakopoulos and Luo (2004). The estimation of $f_{\mathbf{Z}}$ in (1), given $\Lambda_{0}$, needs the estimation of $(N+1)$ local conditional probability density functions (cpdfs), as explained above; furthermore, the decomposition of $f_{\mathbf{Z}}$ in not unique. Then, the cpdfs can be estimated using the well-known sequential conditional simulation algorithm (Alabert 1987; Journel and Alabert 1989; Journel 1994) which follows the well-known sequence: (1) randomly choose the spatial location of a node $x_{i}$ to be simulated; (2) estimate $f_{Z_{i}}\left(z_{i} / \Lambda_{i}\right)$; (3) draw a value 
Fig. 1 An unknown value is at the location $x_{0}$, and the values at the locations $x_{1}, x_{2}, \ldots, x_{n}$ in the neighborhood of $x_{0}$ are assumed to be known

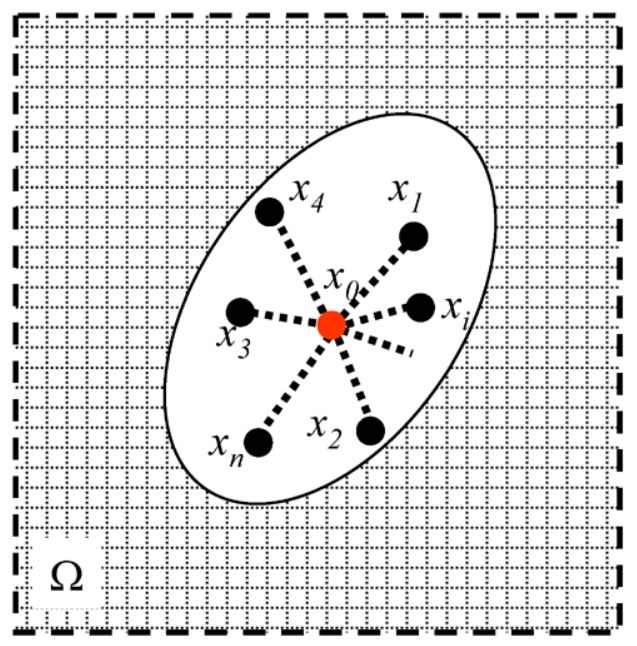

$z_{i}$ from $f_{Z_{i}}\left(z_{i} / \Lambda_{i}\right)$, which becomes a conditioning data for all subsequent drawings; and (4) return to Step (1) until all nodes have been visited using a random path.

For the reason of simplicity and without loss of generality, assume that $x_{0}$ is the first node visited and its neighbors are found within a certain neighborhood, as shown in Fig. 1 . The cpdf $f_{Z_{0}}$, given $\Lambda_{0}$, is defined by

$$
f_{Z_{0}}\left(z_{0} / \Lambda_{0}\right)=\frac{f_{\mathbf{Z}}(\mathbf{z})}{f_{\overline{\mathbf{Z}_{\mathbf{0}}}}\left(\overline{\mathbf{z}_{\mathbf{0}}}\right)},
$$

where $\overline{\mathbf{z}_{\mathbf{0}}}=\left(z_{1}, \ldots, z_{n}\right), \overline{\mathbf{Z}_{\mathbf{0}}}=\left(Z_{1}, \ldots, Z_{n}\right)$, and its marginal density $f_{\overline{\mathbf{Z}_{\mathbf{0}}}}$ is given by

$$
f_{\overline{\mathbf{Z}_{\mathbf{0}}}}\left(\overline{\mathbf{z}_{\mathbf{0}}}\right)=\int_{D} f_{\mathbf{Z}}(\mathbf{z}) d x_{0} .
$$

In the above equations, $D$ denotes the domain of definition of $f_{Z_{0}}$. The estimation of $f_{Z_{0}}$, using (2), requires the approximation of the joint probability density $f_{\mathbf{Z}}$ and the marginal density $f_{\overline{\mathbf{Z}_{\mathbf{0}}}}$. However, the density $f_{\overline{\mathbf{Z}_{\mathbf{0}}}}$ can theoretically be derived from $f_{\mathbf{Z}}$ by integrating along the support of $Z_{0}$. Then, only the estimation of $f_{\mathbf{Z}}$ is sufficient. The aim of the present work is to derive, given $\Lambda_{0}=\left\{Z_{\alpha}=z_{\alpha}, \alpha=1, \ldots, n\right\}$, an estimation of $f_{Z_{0}}$ in the general case, without transforming the conditioning data as, for example, made by the Gaussian-related or indicator-based algorithms. In this paper, we utilize the high-dimensional Legendre polynomials combined with highorder spatial cumulants to derive analytical expressions to the local conditional probability density functions $f_{Z_{i}}\left(z_{i} / \Lambda_{i}\right)$. The method herein developed and its related details are discussed next.

\subsection{Approximation of a Joint Probability Density using Legendre Series}

The determination of a joint PDF, given its cumulants up to order $n$, is a well-known problem, that is, the cumulants problem (Kendall and Stuart 1977) which has been 
Fig. 2 Legendre polynomials up to order 4

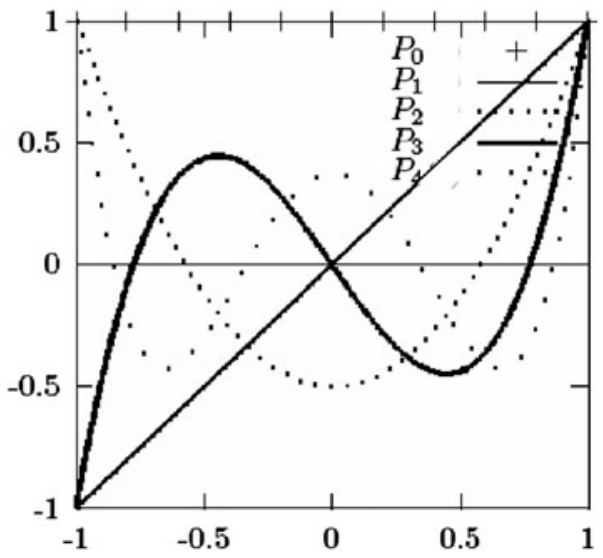

studied extensively from a theoretical point of view. Examples of solving this type of problem can be found in Edgeworth (1905, 1907), Daniels (1954), Lebedev (1965), Welling (1999), Gaztanaga et al. (2000). The approximation used here is based on Legendre series (Lebedev 1965; Liao and Pawlak 1996; Yap and Paramesran 2005; Hosny 2007) with coefficients calculated in terms of high-order spatial cumulants. The method is first reviewed for the univariate case; subsequently, the approximation developed for the general multivariate case is introduced.

\subsubsection{One Dimension}

A square-integrable and real piecewise smooth function $f$ defined on $D=[-1,1]$ can be formally written in a series of Legendre polynomials

$$
f(z)=\sum_{m=0}^{\infty} L_{m} \frac{P_{m}(z)}{\left\|P_{m}\right\|}
$$

where $P_{m}(z)$ is the $m$ th-order Legendre polynomials (Fig. 2), with norm $\left\|P_{m}\right\|$, defined as in Lebedev (1965), Spiegel (1968).

$$
P_{m}(z)=\frac{1}{2^{m} m !}\left(\frac{d}{d z}\right)\left[\left(z^{2}-1\right)^{m}\right]=\sum_{i=0}^{m} a_{i, m} z^{i}, \quad \text { and } \quad-1 \leq z \leq 1 .
$$

The Legendre polynomials $P_{m}(z)$ obey the following recursive relation

$$
P_{m+1}(z)=\frac{2 m+1}{m+1} z P_{m}(z)-\frac{m}{m+1} P_{m-1}(z),
$$

where $P_{0}(z)=1, P_{1}(z)=z$, and $m \geq 1$. The set of Legendre polynomials $\left\{P_{m}(z)\right\}_{m}$ forms a complete orthogonal basis set on the interval $[-1,1]$. The orthogonality property is defined as

$$
\int_{D} P_{m}(z) P_{n}(z) d x= \begin{cases}0, & m \neq n, \\ \frac{2}{2 m+1}, & m=n .\end{cases}
$$


The discrete Legendre polynomials also satisfy

$$
\sum_{i=1}^{k} P_{m}\left(z_{i}\right) P_{n}\left(z_{i}\right) \Delta z=\frac{2}{2 m+1} \delta_{m n}, \quad \forall m, n \geq 0,
$$

where $\Delta z=z_{i}-z_{i-1}=2 / k$ is a space step, $k$ is the number of steps, $\left\{z_{i}\right\}$ is a uniform discretization of $[-1,1]$, and $\delta_{m n}$ is the delta Dirac function. To avoid numerical instability in polynomial computation, we normalized the Legendre polynomials by utilizing the square norm. The set of normalized Legendre polynomials is defined as

$$
\bar{P}_{m}(z)=\sqrt{\frac{2 m+1}{2}} P_{m}(z)
$$

In this case, the orthogonality condition given in (7) becomes

$$
\sum_{i=1}^{k} \bar{P}_{m}\left(z_{i}\right) \bar{P}_{n}\left(z_{i}\right) \Delta z=\delta_{m n}, \quad \forall m, n \geq 0 .
$$

The coefficients $L_{m}$ in (4) of the Legendre series, the so-termed Legendre cumulants, can be determined using the orthogonality property in (7) as

$$
L_{m}=\int_{D} \bar{P}_{m}(z) f(z) d z=g_{m}\left(c_{i}\right), \quad i=0, \ldots, m \text { and } m=0,1,2, \ldots,
$$

where $c_{i}$ is the $i$ th-order cumulant of $f$. The expression of the right-hand side function $\mathrm{g}_{m}$ and other details about cumulants are given in Appendix B. Theoretically, the series (1), with coefficients $L_{m}$ calculated from (5), converges to $f(z)$ at every continuity point of $f(z)$, as demonstrated by Lebedev (1965). Finally, if only cumulants of order smaller than or equal to $\omega$ are given, then the function $f(z)$ in (1) can be approximated as follows

$$
f(z) \approx \tilde{f}_{\omega}(z)=\sum_{m=0}^{\omega} L_{m} \bar{P}_{m}(z)
$$

\subsubsection{Two Dimensions}

Taking the orthogonally principle into consideration, a piecewise function $f\left(z_{0}, z_{1}\right)$ can be written over the square $D^{2}=[-1,1] \times[-1,1]$ as

$$
f\left(z_{0}, z_{1}\right)=\sum_{m=0}^{\infty} \sum_{n=0}^{\infty} L_{m, n} \bar{P}_{m}\left(z_{0}\right) \bar{P}_{n}\left(z_{1}\right)
$$

where the coefficients $L_{m, n}$ are given by

$$
\begin{aligned}
L_{m, n} & =\int_{D^{2}} \bar{P}_{m}\left(z_{0}\right) \bar{P}_{n}\left(z_{1}\right) f\left(z_{0}, z_{1}\right) d z_{0} d z_{1} \\
& =g_{m, n}\left(c_{i j}\right), \quad i, j=0, \ldots, m \text { and } m=0,1,2, \ldots
\end{aligned}
$$


If only the cumulants of order smaller or equal to $\omega$ are given, then the function $f\left(z_{0}, z_{1}\right)$ can be approximated by a continuous function which is a truncated series,

$$
f\left(z_{0}, z_{1}\right) \approx \tilde{f}_{\omega}\left(z_{0}, z_{1}\right)=\sum_{m=0}^{\omega} \sum_{n=0}^{m} L_{m-n, n} \bar{P}_{m-n}\left(z_{0}\right) \bar{P}_{n}\left(z_{1}\right) .
$$

\subsubsection{N Dimensions}

The same procedure is followed to write a piecewise smooth $N$-dimensional function $f\left(z_{0}, z_{1}, \ldots, z_{N}\right)$ over $D^{N+1}=[-1,1]^{N+1}$ by

$$
\begin{aligned}
& f\left(z_{0}, z_{1}, \ldots, z_{N}\right) \\
& \quad \approx \tilde{f}_{\omega}\left(z_{0}, z_{1}, \ldots, z_{N}\right) \\
& \quad=\sum_{i_{0}=0}^{\omega} \ldots \sum_{i_{N-1}=0}^{i_{N-2}} \sum_{i_{N}=0}^{i_{N-1}} L_{\bar{i}_{0}, \ldots, \bar{i}_{N-1}, i_{N}} \bar{P}_{\bar{i}_{0}}\left(z_{1}\right) \cdots \bar{P}_{\bar{i}_{N-1}}\left(z_{N-1}\right) \bar{P}_{i_{N}}\left(z_{N}\right),
\end{aligned}
$$

where $\bar{i}_{k}=i_{k}-i_{k+1}$ for $k<N$. The calculation of the coefficients $L_{i_{1}, \ldots, i_{N-1}, i_{N} \text { will }}$ be pointed out in the next section. As explained above, an approximation of the cpdf $f_{Z_{0}}\left(z_{0} / \Lambda_{0}\right)$ can be derived if the joint probability density $f_{\mathbf{Z}}$ in (2) is estimated. However, the expression in (14) can be used to approximate $f_{\mathbf{Z}}$. Then, the cpdf $f_{Z_{0}}$, given $\Lambda_{0}=\left\{d_{n}\right\}$, can be written as

$$
\begin{aligned}
f_{Z_{0}}\left(z_{0} / \Lambda_{0}\right) & =\frac{f_{\mathbf{Z}}(\mathbf{z})}{f_{\overline{\mathbf{Z}_{\mathbf{0}}}}\left(\overline{\mathbf{z}_{\mathbf{0}}}\right)}=\frac{1}{\int_{D} f_{\mathbf{Z}}(\mathbf{x}) d z_{0}} f_{\mathbf{Z}}\left(z_{0}, z_{1}, \ldots, z_{n}\right) \\
& \approx \tilde{f}_{Z_{0}, \omega}\left(z_{0} / \Lambda_{0}\right) \\
& =\frac{1}{\int_{D} f_{\mathbf{Z}}(\mathbf{x}) d z_{0}} \sum_{i_{0}=0}^{\omega} \ldots \sum_{i_{N-1}=0}^{i_{N-2}} \sum_{i_{N}=0}^{i_{N-1}} \bar{L}_{\bar{i}_{0}, \bar{i}_{1}, \ldots, \bar{i}_{N-1}, i_{N}} \bar{P}_{\bar{i}_{0}}\left(z_{0}\right),
\end{aligned}
$$

where $\bar{L}_{\bar{i}_{0}, \ldots, \bar{i}_{N-1}, i_{N}}=L_{\bar{i}_{0}, \ldots, \bar{i}_{N-1}, i_{N}} \bar{P}_{\bar{i}_{1}}\left(z_{1}\right) \cdots \bar{P}_{\bar{i}_{N-1}}\left(z_{N-1}\right) \bar{P}_{i_{N}}\left(z_{N}\right)$. Here, we note that $\left\{z_{1}, \ldots, z_{n}\right\}$ are given, and they are the values of the samples around $x_{0}$. Equation (15) is the main expression which is used to approximate local pdfs.

Generally speaking, and to further comment on the cpdf estimation procedure above, note that a sequence of estimators for a parameter $a$ is said to be (asymptotically) consistent if this sequence converges in probability to $a$. The proof of consistency differs from method to method. For example, in the case of sequential Gaussian simulation algorithms, the covariance of two variables at two locations separated by a distance $h$ is inferred from a variogram model. The variogram model also ensures that the covariance tends to zero as the distance tends to infinity. Then, the covariance matrix is inverted to estimate the parameters of a Gaussian distribution; this matrix has to be symmetric positive definite. However, if the approximation developed in this paper asymptotically converges to the true distribution (Hille 1926; Lebedev 1965), then there is no need to invert cumulant matrices. Cumulants are not modeled and are 
calculated here experimentally. This is analogous, only in terms of calculation of the high-order moments, to the snesim algorithm (Strebelle 2002). Then, consistency for the developed approach is realized by the asymptotic convergence.

\subsection{Approximation of the Coefficients of the Legendre Series using Spatial Cumulants}

Consider the sample case of estimating the cpdf of $Z_{0}$ using the first four samples at the locations $x_{1}, x_{2}, x_{3}$, and $x_{4}$ in Fig. 1. The coefficients of the Legendre series in (15) combine different high-order spatial cumulants. For example, when $\omega=2$, the coefficient $L_{22111}$ is given by

$$
\begin{aligned}
L_{22111} & =\int_{D^{5}} \bar{P}_{2}\left(z_{0}\right) \bar{P}_{2}\left(z_{1}\right) \bar{P}_{1}\left(z_{2}\right) \bar{P}_{1}\left(z_{3}\right) \bar{P}_{1}\left(z_{4}\right) f\left(z_{0}, z_{1}, z_{2}, z_{3}, z_{4}\right) d \mathbf{z} \\
& =g_{22111}\left(c_{j_{0}, j_{1}, j_{2}, j_{3}, j_{4}}\right), \quad \text { and } \quad\left\{j_{0}, j_{1}, j_{2}, j_{3}, j_{4}\right\} \subseteq\{0,1,2\}
\end{aligned}
$$

where $c_{j_{0}, j_{1}, j_{2}, j_{3}, j_{4}}=\operatorname{cum}\left(Z_{0}^{j_{0}}, Z_{1}^{j_{1}}, Z_{2}^{j_{2}}, Z_{3}^{j_{3}}, Z_{4}^{j_{4}}\right)$ is a cumulant of order $n=j_{0}+$ $j_{1}+j_{2}+j_{3}+j_{4}$ of the true local conditional distribution.

A high-order cumulant is inferred from a TI combined with samples. The procedure used can be described as follows:

1. Define a spatial template (ST) using $x_{0}$ and its neighbors as shown in Fig. 1. We first define different directions $\left(\vec{d}_{i}\right)$ pointed from $x_{0}$ toward the neighbors $x_{i}$. These directions are supported by different angles $\left\{\alpha_{i}\right\}$ measured positive from the north. Denote by $h_{i}$ the lag distance between $x_{0}$ and $x_{i}$.

2. Search all the replicates by scanning a given TI with samples with the ST defined in Step 1. The set of replicates obtained is given by (considering a spatial location $x$ as a reference)

$$
\begin{aligned}
& T^{h_{1}, h_{2}, h_{3}, h_{4}}\left(h_{1}, h_{2}, h_{3}, h_{4}, \alpha_{1}, \alpha_{2}, \alpha_{3}, \alpha_{4}\right) \\
&=\left\{\left(x, x+h_{1}, x+h_{2}, x+h_{3}, x+h_{4}\right) /\left\{x, x+h_{i}, i \text { from } 1 \text { to } 4\right\}\right. \\
&\text { is a set of points in the original distribution }\},
\end{aligned}
$$

where the point $x+h_{i}$ is implicitly calculated from $x+h_{i} \vec{d}_{i}$, and $\vec{d}_{i}$ is the unit vector which determines the direction of $h_{i}$.

3. Use the replicates in (17) to experimentally calculate the cumulants as, for example, shown in Appendix A. Details on the calculation of spatial cumulants can be found elsewhere (Dimitrakopoulos et al. 2010; Mustapha and Dimitrakopoulos 2010).

The approximation given by (5) may generate negative values; for that reason, let us discuss the three different cases presented in Fig. 3. The Legendre series approximation is always positive for cases 1 and 2, where $y=\inf \{f(x)\} \geq \varepsilon$, and $\varepsilon$ is a small positive real number. Case 3 shows that $y=\inf \{f(x)\}=0$; let $x_{0}$ be the corresponding $x$-coordinate of $y$. The Legendre series may be negative in a small interval centered at $x_{0}$; to solve this problem, different numerical techniques may be used: 

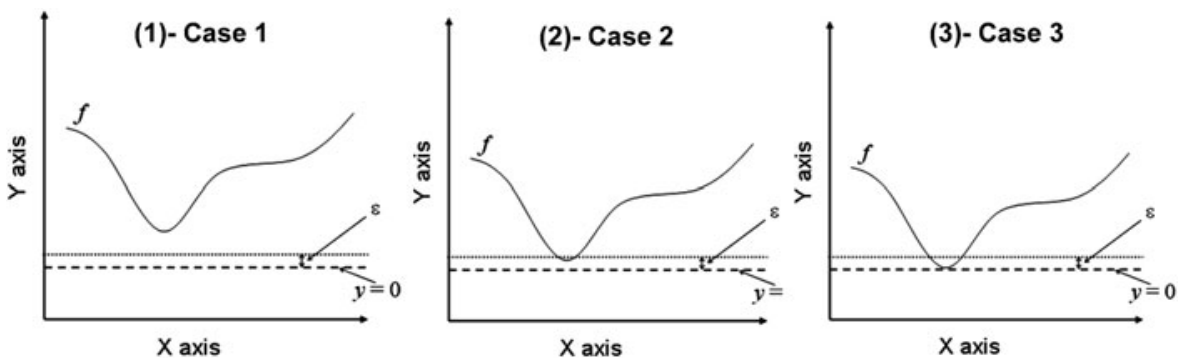

Fig. 3 Density profile. Three different cases are distinguished with respect to a small positive real number $\varepsilon$

1. Correct the density approximation around $x_{0}$ by interpolation (Wilson and Wragg 1973).

2. Introduce $\hat{f}=\max \{\tilde{f}, 0\}$; it is evident that $|\hat{f}-f| \leq|\tilde{f}-f|$. Here, the small negative part can be neglected, and $\hat{f}$ is scaled by the area of the positive region to sum to one.

In this paper, the first solution is adopted; however, the second solution was tested and showed very similar results to the first solution.

\subsection{A High Quality Approximation}

The truncated Legendre series at the order $n$ tends to the true distribution, and the quality of the approximation stabilizes after certain order. For example, Fig. 4 shows that the Legendre series of orders 6, 12, and 25 fit very well the true distribution. Moreover, the Legendre series of orders 12 and 25 are too close and provide about the same approximation.

Assume that $c_{i}$ (resp., $m_{i}$ ), $i=1, \ldots, n$, are the first $n$ cumulants (resp., $n$ moments) of the true distribution $f$. Each cumulant order provides certain information that may not be seen using other cumulants of other orders. Mustapha and Dimitrakopoulos (2010) show that additional information describing complex geological patterns may be obtained by increasing the order of cumulants; however, some other information may only be detected by the cumulants and/or moments of lower orders. For example, if a random variable takes values in ]0, 1[, then all its high-order moments may tend to zero after certain order. Then, it is very difficult to derive a good approximation of $f$ using only one high-order cumulant $\left(c_{n}\right)$ or moment $\left(m_{n}\right)$. Consider, for example, the Legendre series of order 12 without using the first three cumulants $c_{1}, c_{2}$, and $c_{3}$. Now, this series does not provide a good approximation of the true distribution, as shown in Fig. 5. Note that the quality of the approximation may decrease more if, for example, $c_{4}$ and other cumulants of order lower than 12 are not used.

Now, from a geological point of view, the quality of the approximation is, first, strongly related to the method used and how this method employs the available data. For example, the snesim algorithm in Strebelle (2002) calculates only one high-order moment that corresponds in (16) to $\left\{j_{0}=1, j_{1}=1, j_{2}=1, j_{3}=1, j_{4}=1\right\}$. Strebelle (2002) shows that the TI, used to infer these moments, determines the main features 
Fig. 4 Approximations using Legendre series

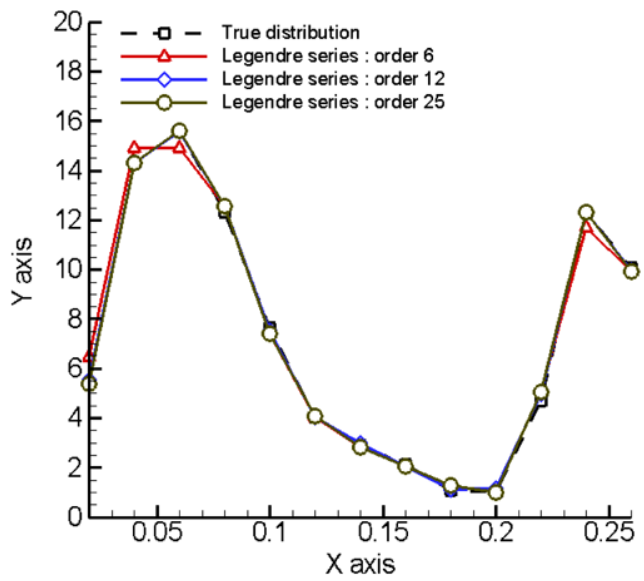

Fig. 5 Approximation using Legendre series and without using the first cumulants $c_{1}, c_{2}$, and $c_{3}$ of the true distribution

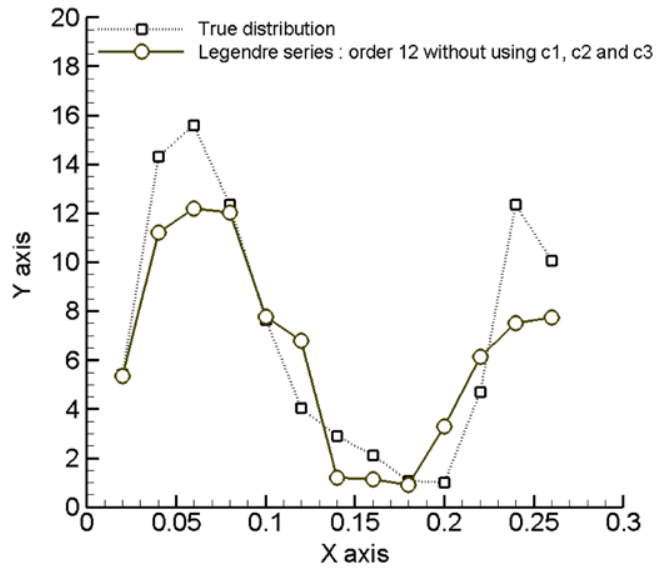

of the simulated images. In addition, he presented critical cases where a conflict may occur if the number of hard data is relatively high $(\sim 200$ data in a $100 \times 100$ pixel image). In conclusion, a poor reproduction of the structures of the true and the TI is obtained. Note that the fact that snesim uses a single normal equation algorithm leads to the data effects not being seen well when using only one high-order moment, as snesim does and was explained earlier. Similar observations are made in actual field applications where substantial available data show that although the spatial statistics of the TI are reproduced, the data statistics may not be (e.g., Volker and Dimitrakopoulos 2007). Similar remarks are generally valid for the filtersim algorithm (Zhang et al. 2006), which uses a limited number of linear filters and may degenerate sample effects.

It follows from the above discussion that the goal of an advanced simulation algorithm is to combine different orders so as to use most of the information available in the available data. This leads to generating data-driven, high-order simulation methods, as opposed to the current TI-driven MP simulation algorithms, and thus overcomes the limitations discussed above. It is important to note that in the present 
Fig. 6 (1) Training image; locations of hard data in (2)
(1)- Training image

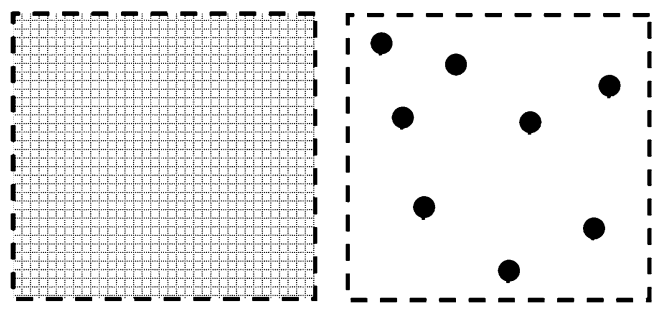

work, moments are replaced by cumulants because cumulants are superior in describing anisotropy of spatial patterns, connectivity of extreme values, and nonlinear spatial architectures. In addition, it is further shown by Dimitrakopoulos et al. (2010) that only a subset of all the cumulant templates has to be computed in order to characterize complex spatial patterns.

\section{A High-order Simulation Method}

This section describes a high-order conditional simulation method (hosim) based on spatial cumulants. A sequential procedure simulating values at unsampled locations that are randomly visited is used here, as explained above. The conditional density expression derived in (15) is employed to estimate the cpdfs. This expression uses Legendre polynomials that are orthogonal on the finite interval $[-1,1]$. Then, the training images and the data values are first scaled to $[-1,1]^{d}$, where $d$ is the dimension of the problem (i.e., $d=1,2$, or 3 ).

The hosim method first combines the TI used and the samples (Fig. 6) to infer high-order spatial cumulants. HOSIM algorithm uses both the data set and the TI to infer the cumulants. The algorithm starts first by searching in the data available. For a given cumulant, if the number of replicates found in the data is less than a given number, the search continues through the TI. Mostly, the lower orders will be reasonably inferred from the data, and the information missed to estimate higherorder cumulants can be borrowed from a TI. Then, a global calculation procedure is performed based on a given maximal template size (TEMP), as shown in Fig. 6(2). This step consists of calculating all the spatial cumulants needed by the Legendre series in (15).

In an MP approach (e.g., the snesim algorithm) the template shape is centered at the unsampled location with branches directed from the center to the data (or the closer $n_{\max }$ data) found within the neighborhood utilized. Similarly, in the hosim algorithm, the global template shape is defined by the unsampled locations and the data within a certain neighborhood. The difference between the two algorithms is that not only the global (or large) template is used by hosim, as done by snesim, but with all the subtemplates (unlike snesim), including those of one branch (unsampled-data: two-point statistics), two branches (usampled-data-data: three-point statistics), and so on (up to $\left(n_{\max }+1\right)$-point statistics). In hosim, the different subtemplates refer to different cumulants or moments. The use of only one template (we mean here the global one) refers to only one high-order moment in the snesim algorithm. 
Fig. 7 Training image in (1). The template in (2) is used for a global calculation of spatial cumulants
(1)- Training image with hard data

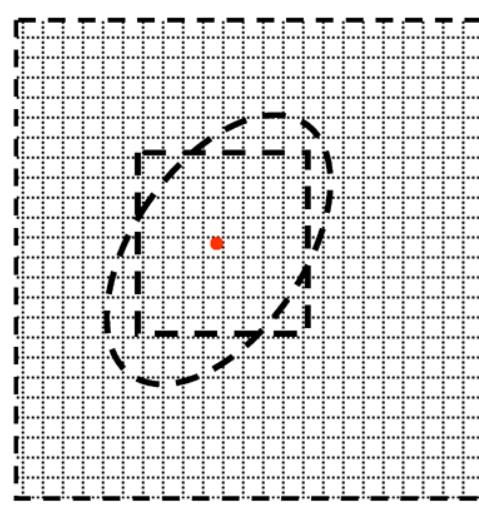

(2)- Template TEMP for global calculation

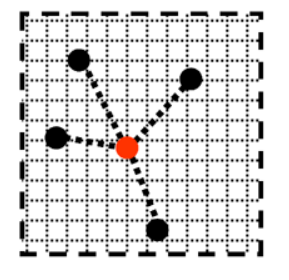

The main steps of the hosim method are as follows:

1. Scan the training image and the sample data (Fig. 7(1)) and store the spatial cumulants calculated using (A.1) in a global tree.

2. Define a random path visiting once all unsampled nodes.

3. Define the template shape $T$ for each unsampled location $x_{0}$ using its neighbors. The conditioning data available within TEMP are then searched (Fig. 7). The highorder spatial cumulants are read from the global tree in Step 1 and are used to calculate the coefficients of the Legendre series, as in (16). These coefficients are used to build the cpdf of $Z_{0}$ using (15).

4. Draw a uniform random value in $[0,1]$ to read from the conditional distribution a simulated value, $Z\left(x_{0}\right)$, at $x_{0}$.

5. Add $x_{0}$ to the set of sample hard data and the previously simulated values.

6. Repeat Steps 4 and 6 for the next points in the random path defined in Step 3.

7. Repeat Steps 3 to 7 to generate different realizations using different random paths.

The random path defined in Step 5 concerns only the unsampled locations. Thus, the realizations obtained after Step 6 honor the conditioning data.

\section{Examples and Comparisons}

The method developed and detailed above is validated by simulating 2D horizontal sections of a 3D fluvial reservoir. The data sets used are available in the Stanford V Reservoir Data Set (Mao and Journel 1999). In one of the examples, we studied the sensitivity of the method to the TI used. We rotated the TI by 90 degree to get another TI. Obviously, we did this because we observed that in the 3D fluvial reservoir, the large channels may completely change directions from NS in a horizontal section to EW in another horizontal section as shown in Fig. 8.

Figure 9(1) shows the exhaustive image to be simulated from different sample data sets. This image is a horizontal section located at $z=8 \mathrm{~m}$. From this section, three different sample data sets have been selected. The first set (DS-1) contains 361 data on a regular sampling grid as shown in Fig. 9(2). The second and third sets are, 
(1)- two-dimensional sections along z

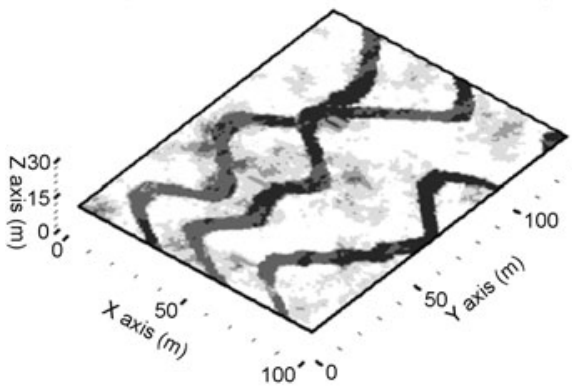

Fig. 8 2D horizontal sections of a fluvial reservoir
(2)- two-dimensional sections along $z$

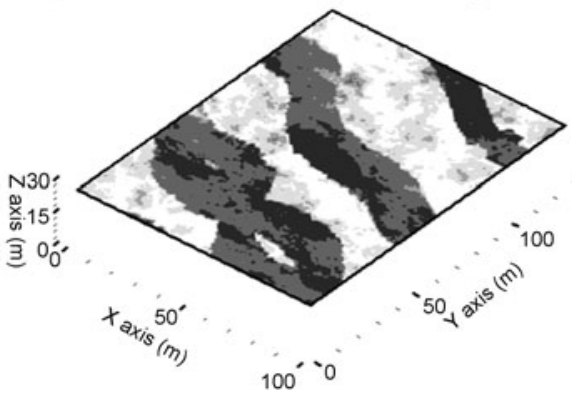

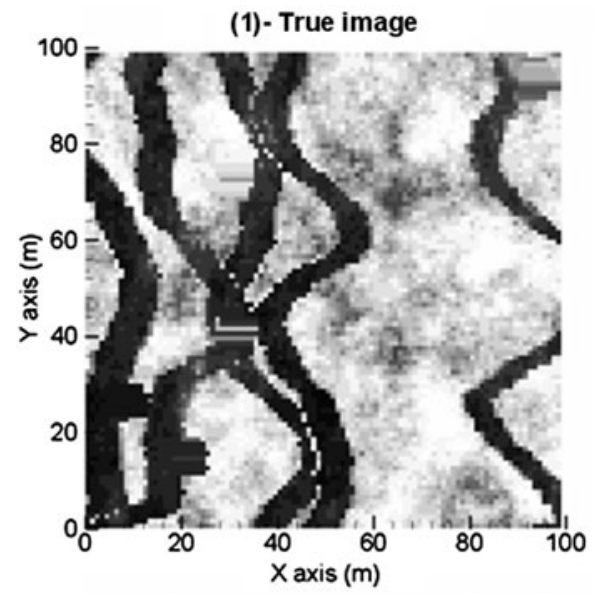

(3)- Sample Data : set 2

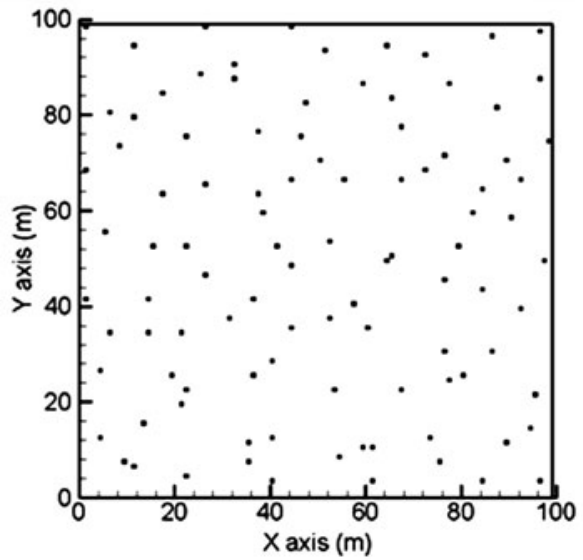

(2)- Sample Data : set 1

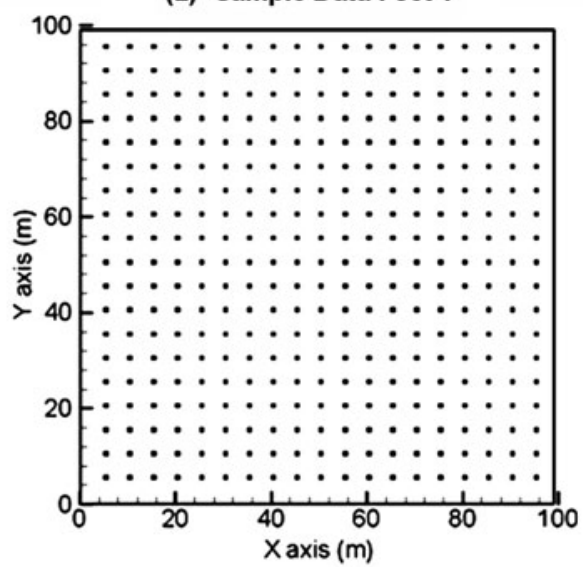

(4)- Sample Data : set 3

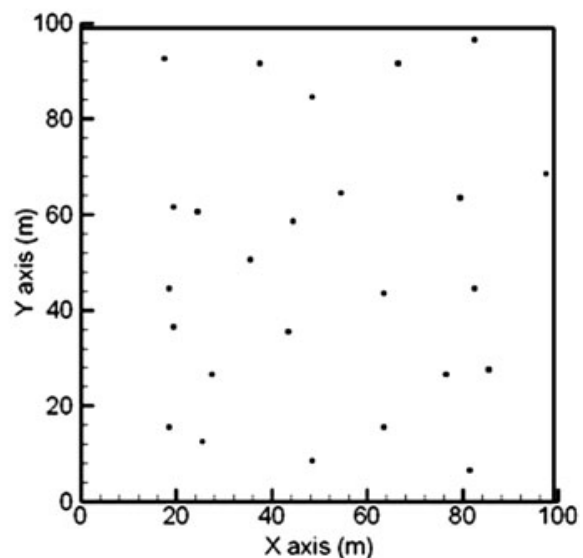

Fig. 9 A horizontal 2D section of a 3D fluvial reservoir. (1) Exhaustive image: true image, (2) DS-1: 361 sample data, (3) DS-2: 85 sample data, (4) DS-3: 25 sample data. (1) is used in all examples to validate hosim algorithm 

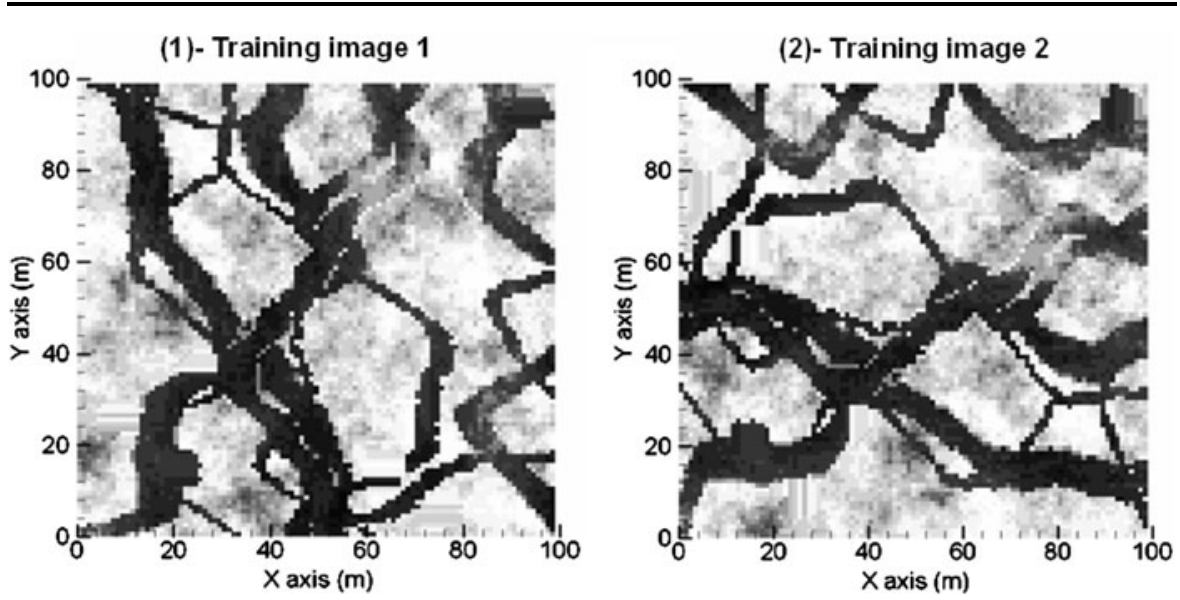

Fig. 10 Two different training images. A 2D horizontal section of the reservoir is on the left (TI-1), and its $90^{\circ}$ rotation is on the right (TI-2)

respectively, generated randomly with 85 (DS-2) and 25 (DS-3) data as shown in Figs. 8(3) and (4).

Each of these data sets is combined with two different training images, TI-1 and TI-2, as shown in Fig. 10, to infer the high-order spatial cumulants that are needed for the estimation of the local pdfs. The TI-1 in Fig. 10(1) is a horizontal section located at $z=7 \mathrm{~m}$ and is rotated in Fig. 10(2) for a sensitivity analysis of hosim to the TIs used. In addition, unconditional realizations and a comparison with other algorithms are presented. All the images used are of size $100 \times 100=10,000$ pixels. Ten realizations are generated using hosim for each of the cases studied, and about 14 nearby data are selected in average for the simulation of any single node. The main features of our approach are illustrated through various examples as shown by the following. All runs are performed on a $3.2 \mathrm{GHz}$ Intel(R) Xeon (TM) PC with 2 GB of RAM.

\subsection{Example 1: Simulation of the Exhaustive Image Using DS-1}

This example consists of reproducing the exhaustive image in Fig. 9(1) using DS-1, and the high-order cumulants are inferred from the exhaustive image. Figure 11 shows two generated realizations; these realizations show that the main channels in the exhaustive image are reproduced. Moreover, the small details in the exhaustive image have been reflected by the realizations, as shown in the zones $80<x<100$ and along $y$ of Figs. 20(1) and (2).

The 2D sections presented here have particular and complex distributions as shown by the bimodal histogram in Fig. 12(1). This figure shows the comparison between the ten (hosim) realizations histograms and the DS-1 histogram. The left and right heads of the data histogram are well reproduced by the realizations. In addition, the realizations reproduced the variograms along the EW and NS directions DS-1 as shown in Figs. 11(2) and (3). 

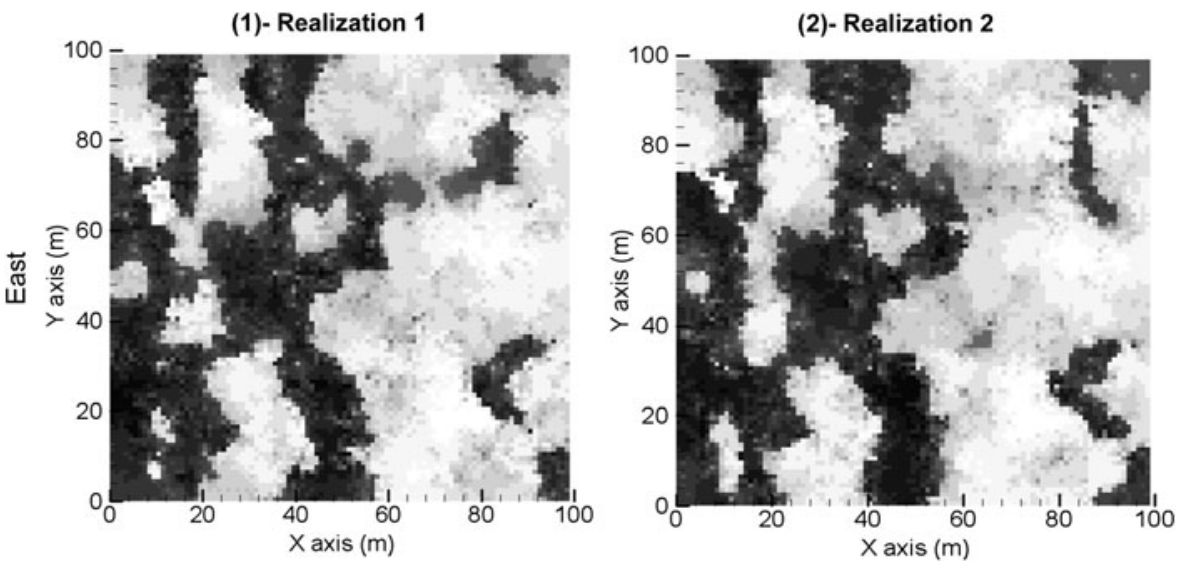

Fig. 11 Realizations (1) and (2) obtained by hosim. Example 1, DS-1
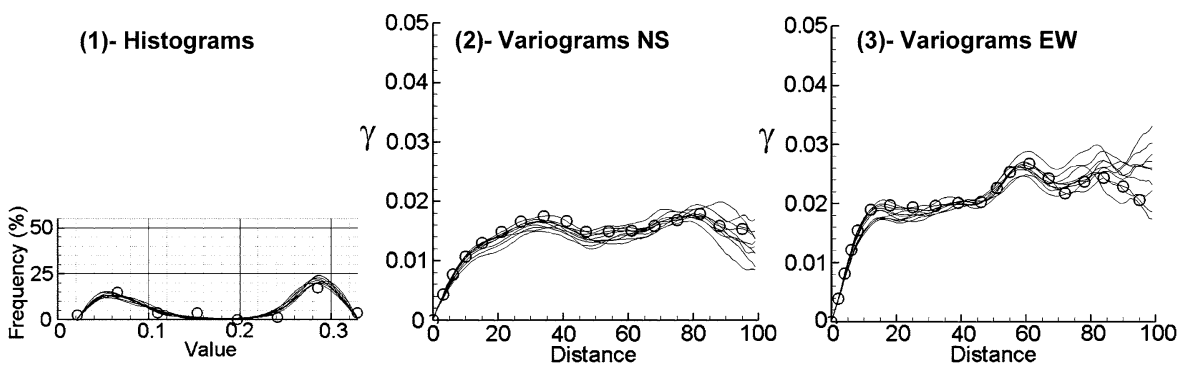

Fig. 12 Histograms (1), NS (2), and EW (3) variograms of 10 hosim realizations. The circles refer to the data set 1 , and the solid lines refer to the realizations. Example 1, DS-1

The proposed method is also validated by comparing the high-order statistics of the DS-1, exhaustive image and the different realizations obtained. For example, the third-order spatial cumulant maps of the exhaustive image, DS-1, realizations (1) and (2), are very close, as shown in Fig. 13. As explained in the above discussion, we stress here that using different cumulants orders in the Legendre series (15) will guarantee the reproduction of not only the histogram and variograms of the sample data, but also their high-order statistics.

Note that the reproduction of thin channels is, in another word, a reconstruction of the very local properties and/or spatial variability of the attribute. In general, this is strongly related to the spatial distribution of the available data. Moreover, hosim is a conditional simulation algorithm and the information borrowed from the TI is conditional to data available in a neighborhood of the simulated point. Then, local information (that is, width of thin channels) can only be described if a certain quantity of information from the data is available; in this case, the image can be completed by the information from the TI. 

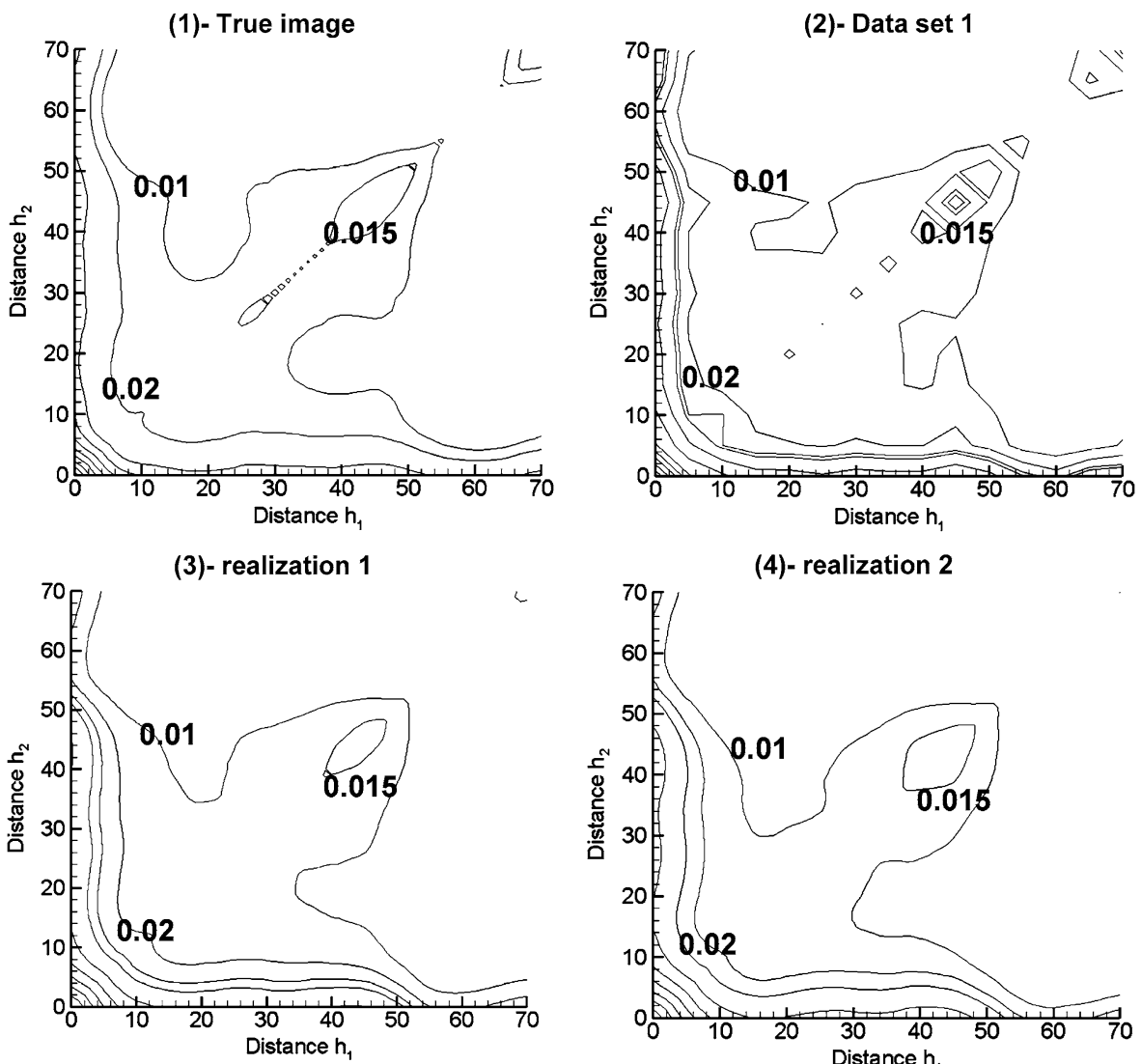

(4)- realization 2

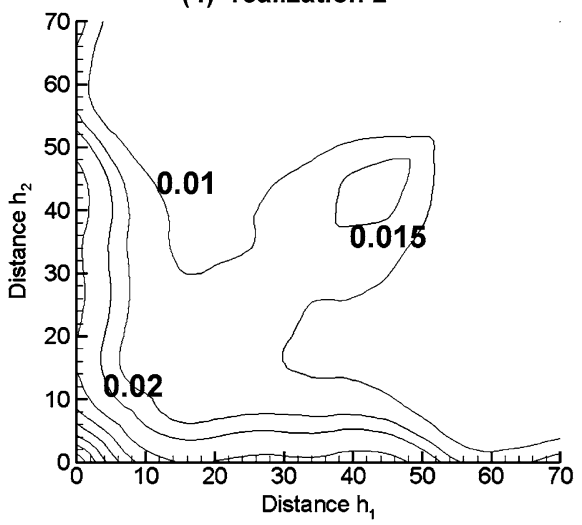

Fig. 13 Third-order spatial cumulant maps of (1) the true image, (2) the hard data set 1, (3) and (4) the realizations 1 and 2, respectively. Values 0.01, 0.015, and 0.020 are the isovalue contours. Example 1, DS-1

\subsection{Example 2: Simulation of the Exhaustive Image Using TI-1, DS-1, and DS-2}

In this example, the TI-1 in Fig. 10(1) is combined with DS-1 and DS-2 to infer the high-order spatial cumulants. First, DS-1 is used, and the realizations generated in example 1 have been repeated. The results are presented in Fig. 14 and are excellent compared to the realizations and the exhaustive image in Example 1. Similarly, the comparison between the histograms, NS and EW variograms, third-order cumulants of hosim realizations, and DS-1 is shown in Figs. 15 and 16, showing excellent results.

Using a smaller number of hard data (DS-2), excellent results are obtained as shown in Fig. 17. The main characteristics of the exhaustive image are reproduced using a sparse data set (about $0.85 \%$ of the total number of points). In addition, excellent results are obtained by comparing the statistics of the realizations and the exhaustive image, as shown in Figs. 18 and 19. 

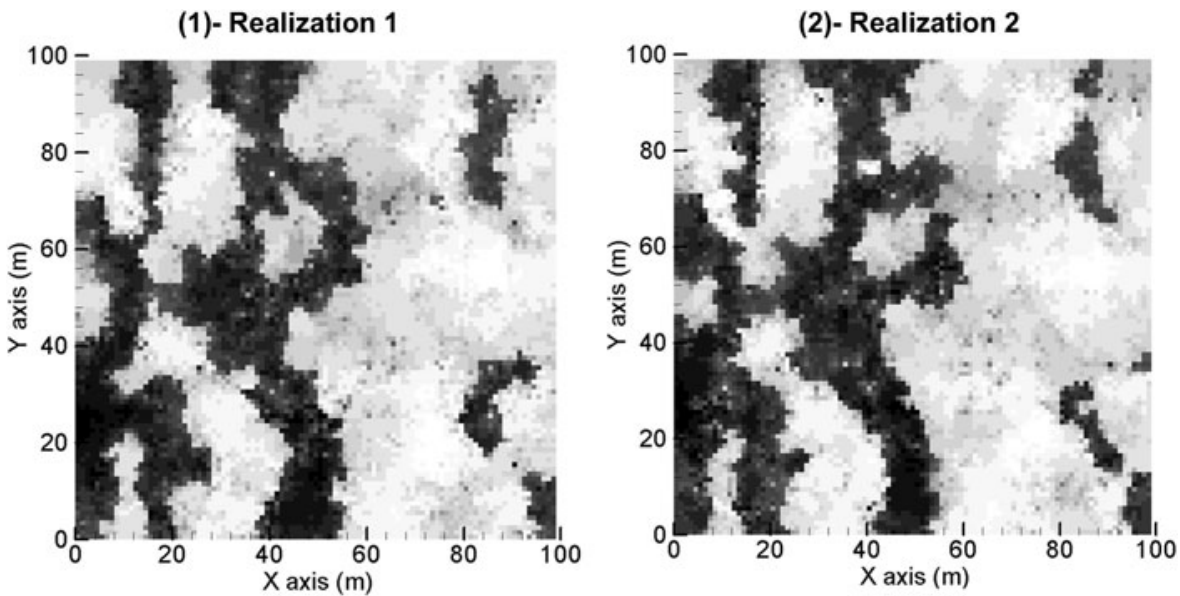

Fig. 14 Realizations (1) and (2) obtained by hosim. Example 2, TI-1, DS-1
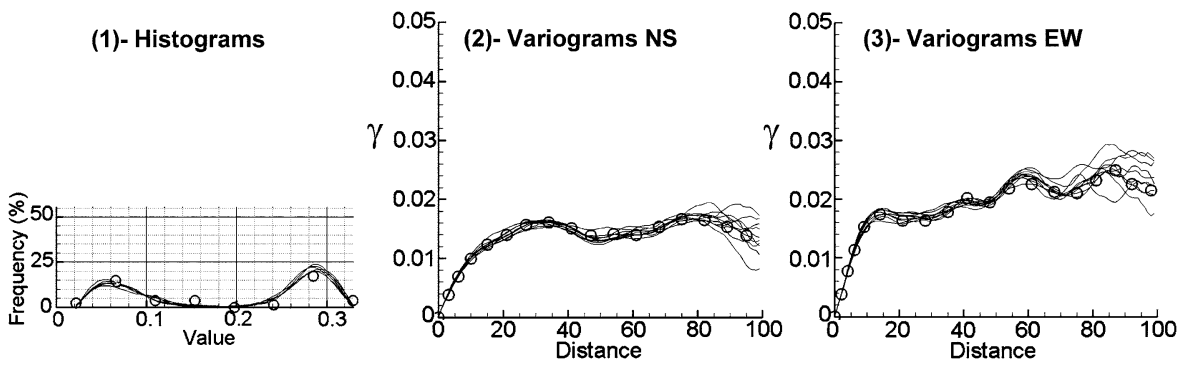

Fig. 15 Histograms (1) and variograms NS (2) and EW (3) of 10 hosim realizations. The circles refer to the data set 1, and the solid lines refer to the realizations. Example 2, TI-1, DS-1

(1)- realization 1

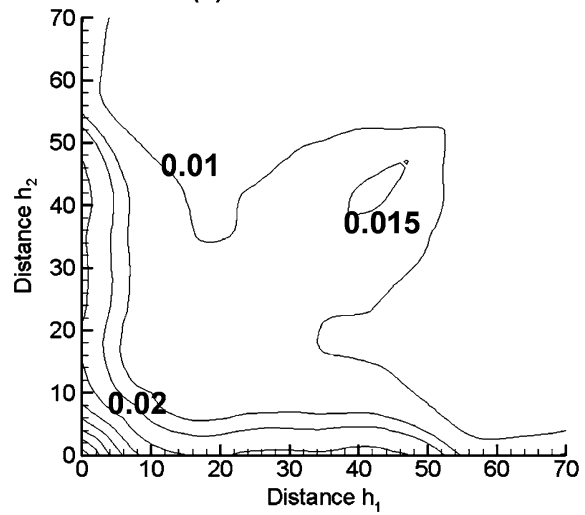

(2)- realization 2

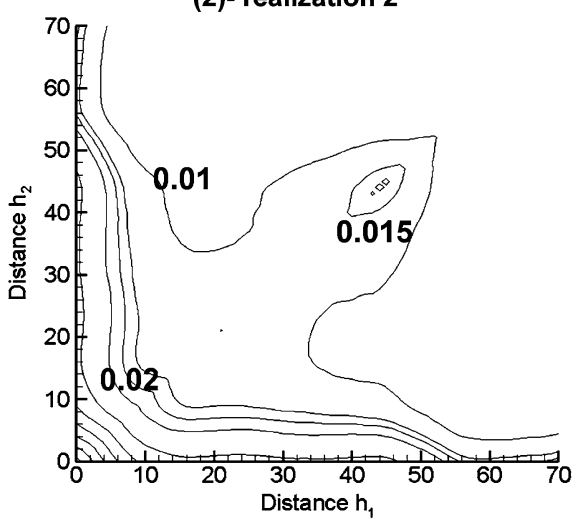

Fig. 16 Third-order spatial cumulant maps of the realizations 1 and 2, respectively. Example 2, TI-1, DS-1 

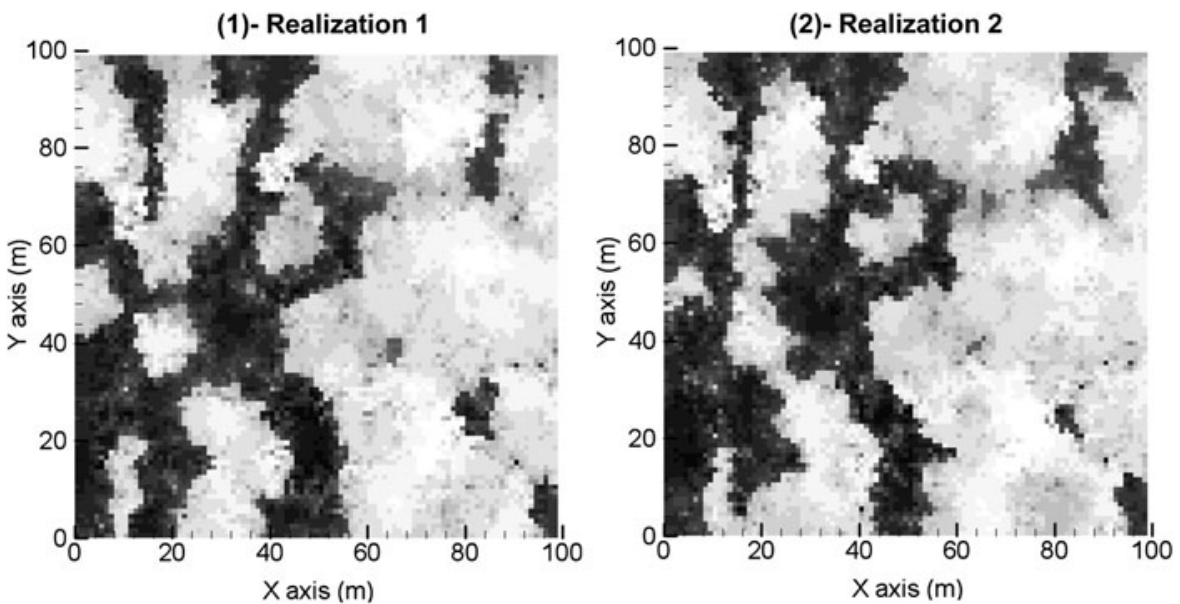

Fig. 17 Realizations (1) and (2) obtained by hosim. Example 2, TI-1, DS-2
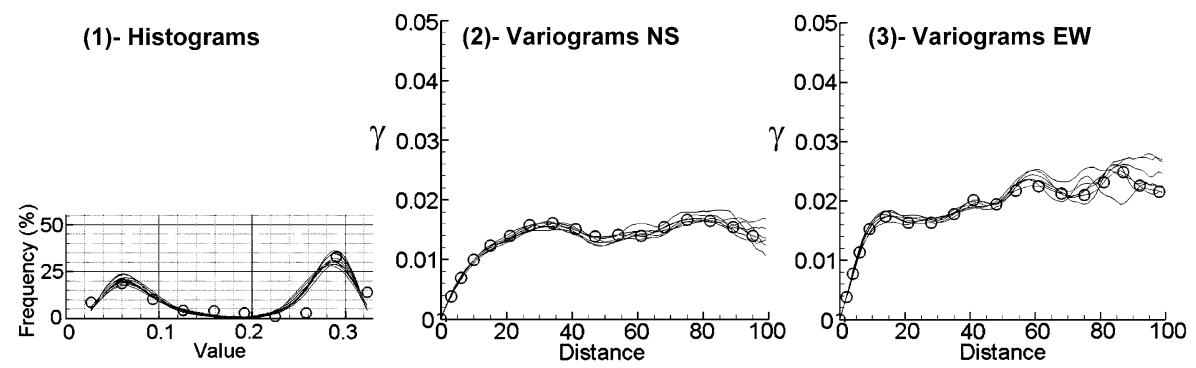

Fig. 18 Histograms (1), NS (2) and EW (3) variograms of 10 hosim realizations. The circles refer to the data set 1, and the solid lines refer to the realizations. Example 2, TI-1, DS-2

(1)- realization 1

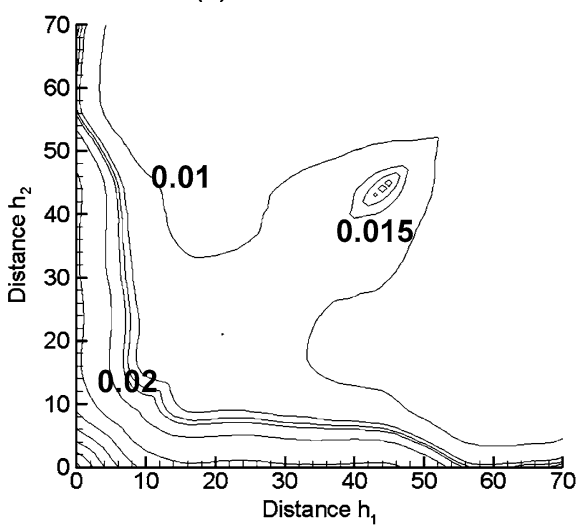

(2)- realization 2

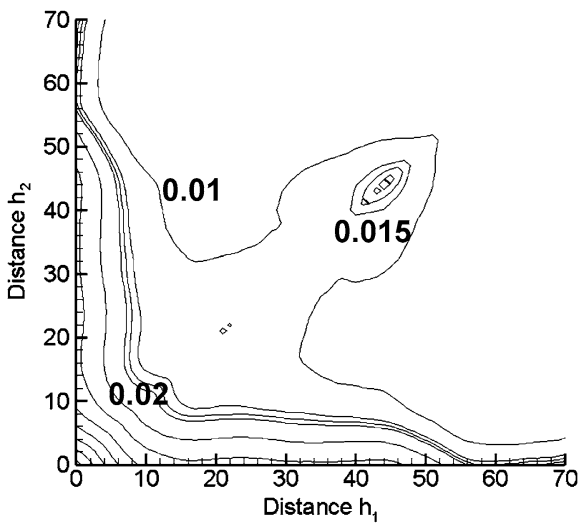

Fig. 19 Third-order spatial cumulant maps of the realizations 1 and 2, respectively. Example 2, TI-1, DS-2 

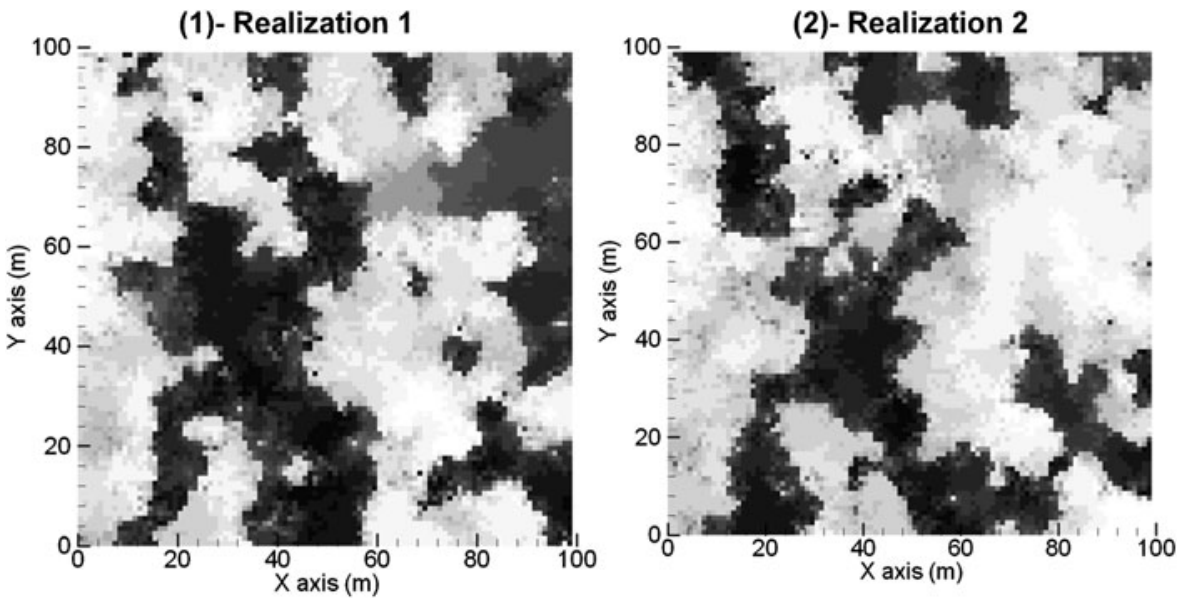

Fig. 20 Unconditional realizations obtained by hosim using TI-1
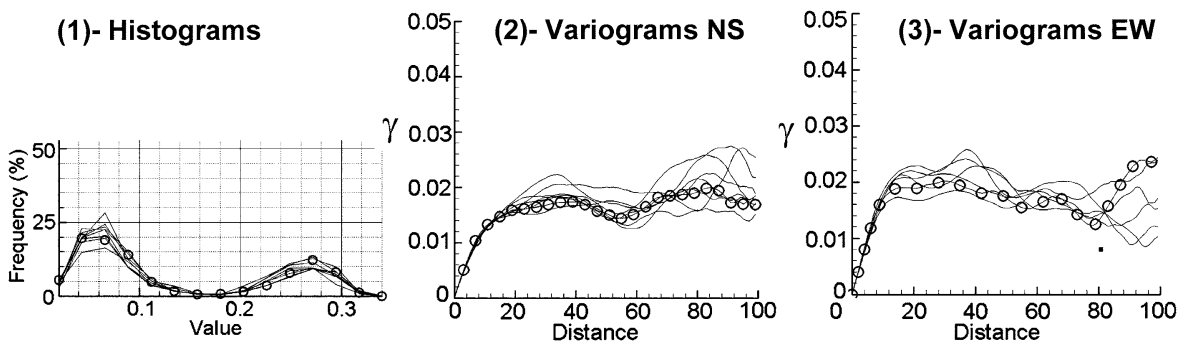

Fig. 21 Histograms (1) and variograms NS (2) and EW (3) of 10 unconditional hosim realizations. The circles refer to TI-1, and the solid lines refer to the realizations

\subsection{Example 3: Unconditional Simulation of the Exhaustive Image Using TI-1}

This example presents unconditional realizations generated with hosim. The training image TI-1 is used to infer the different high-order spatial cumulants. Figure 20 depicts two realizations and shows that the realizations reproduce the main features of TI-1. The absence of data lets the TI's underlying characteristics dominate. Comparisons of statistics show that the statistics of TI-1 are reproduced by the realizations, as shown in Fig. 21.

Up to this point, the examples have shown that the proposed method is data-driven, and as the data decreases, their effect on the simulated realizations decreases, leading to finally generating unconditional realizations.

\subsection{Example 4: Sensitivity of the Simulation Algorithm to the TI Used}

The TI controls the generation of realizations when the number of samples is too small and vice versa. Then, the quantity of information borrowed from a TI decreases for a corresponding increase in the number of samples. In this context, the effects 

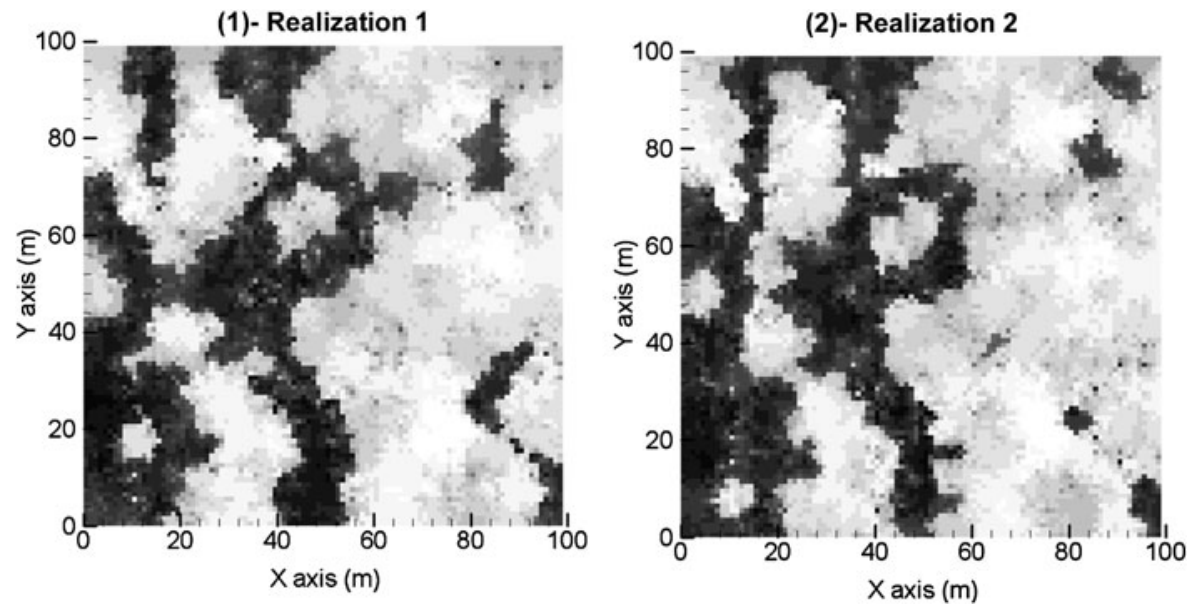

Fig. 22 Realizations (1) and (2) obtained by hosim. Example 3, TI-2, DS-2

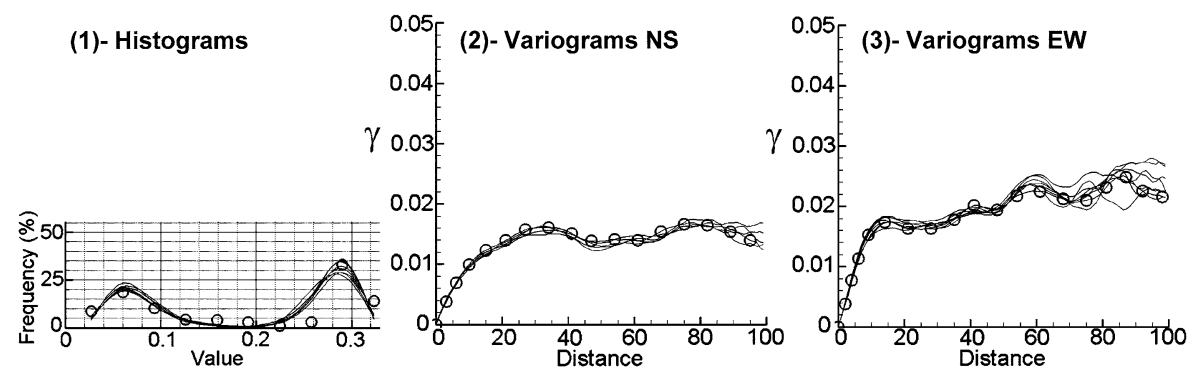

Fig. 23 Histograms (1) and variograms NS (2) and EW (3) of 10 hosim realizations. The circles refer to the data set 1, and the solid lines refer to the realizations. Example 3, TI-2, DS-2

of the number of data and TIs on the final realizations obtained using hosim are examined. In the above examples, the channels in the true image and in the TIs have the same orientation. However, Fig. 8 shows two different channel configurations orientations along one direction of the fluvial reservoir. To cover this case, TI-1 is rotated by $90^{\circ}$ to obtain TI- 2 in Fig. 10(2). First, using TI- 2 and DS-2, the realizations in Fig. 22 are obtained. These results still show a good reproduction of the exhaustive image. The statistics of the realizations are extremely close to the sample data and exhaustive image as shown in Figs. 23 and 24.

Now, using TI-2 and DS-3, a clear conflict between the effects of the samples and the training image is obtained, as shown in Fig. 25(2). Here, the channels deviated and are now more related to the heterogeneities of the training image. In contrast, the TI-1 combined with DS-3 provided good approximation of the channels in the exhaustive image, as shown in Fig. 25(1).

In relation to the discussion in the section above, first the estimation of the highorder cumulants is constrained by the knowledge of certain values of the true distribution. In that discussion, we can clearly see that the estimation of the moments and/or 
(1)- realization 1

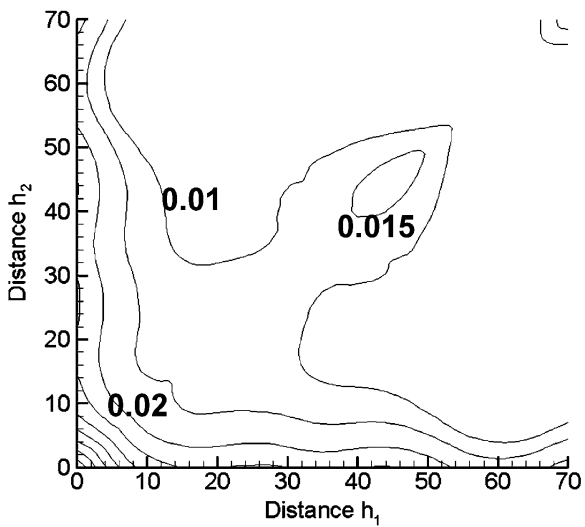

(2)- realization 2

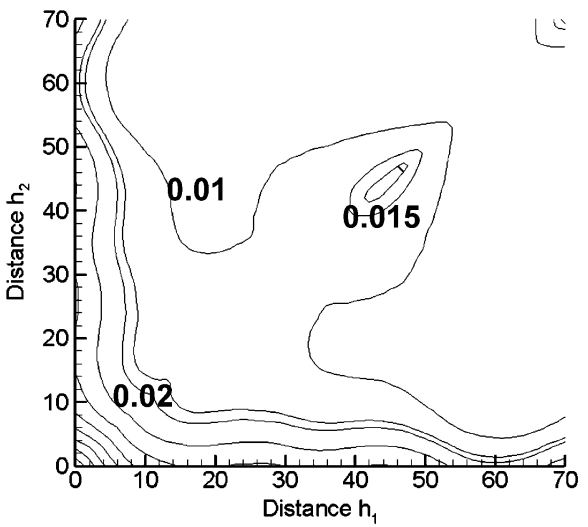

Fig. 24 Third-order spatial cumulant maps of the realizations 1 and 2, respectively. Example 3, TI-2, DS-2
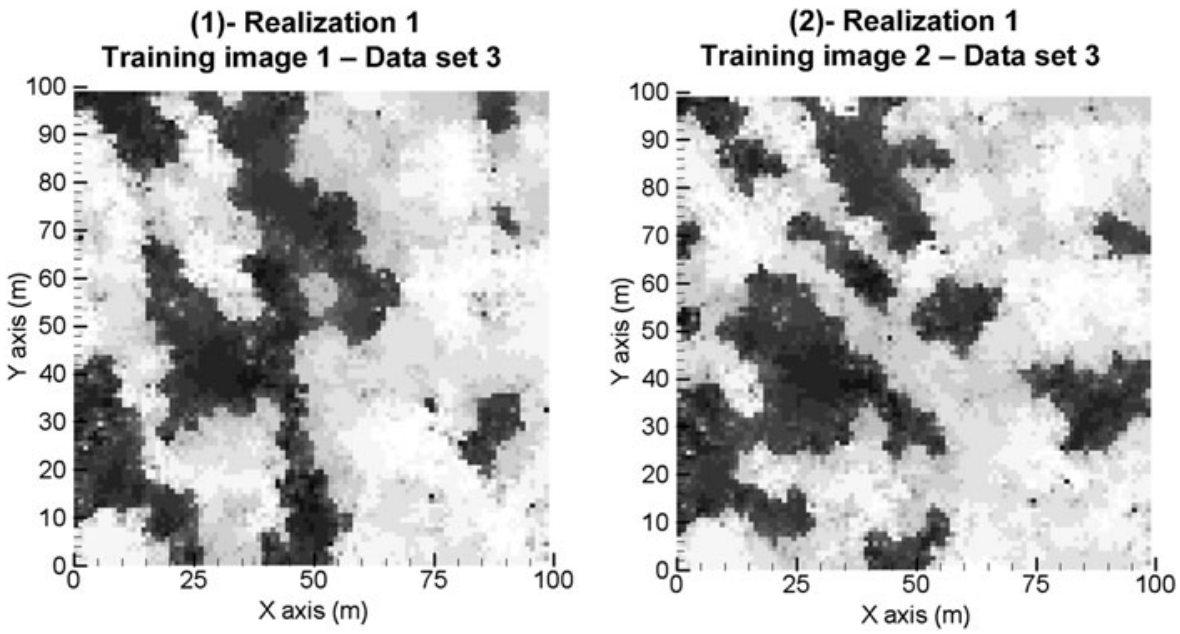

Fig. 25 Realizations obtained by hosim using TI-1 (left) and TI-2 (right) with DS-3

cumulants of an unknown distribution $f$ can be better inferred, using, for example, a Riemann integration method, if the number of points at which $f$ is known increases. Now, if the cumulants of $f$ are only inferred from another auxiliary distribution (i.e., here the TI), the final realizations, as shown in snesim for example, will strongly depend on the TI used. Combining a TI with known values of the distribution (i.e., the sample data) may first adjust the auxiliary distribution and then modify its statistics to be closer to the statistics of the true distributions. In this case, the TI will have less of an effect on the generated realizations. Moreover, the use of different orders of cumulants contributes to a better description of the connectivity of the sample values. Consequently, the effects of the TI on the realizations decrease. In this context, the 

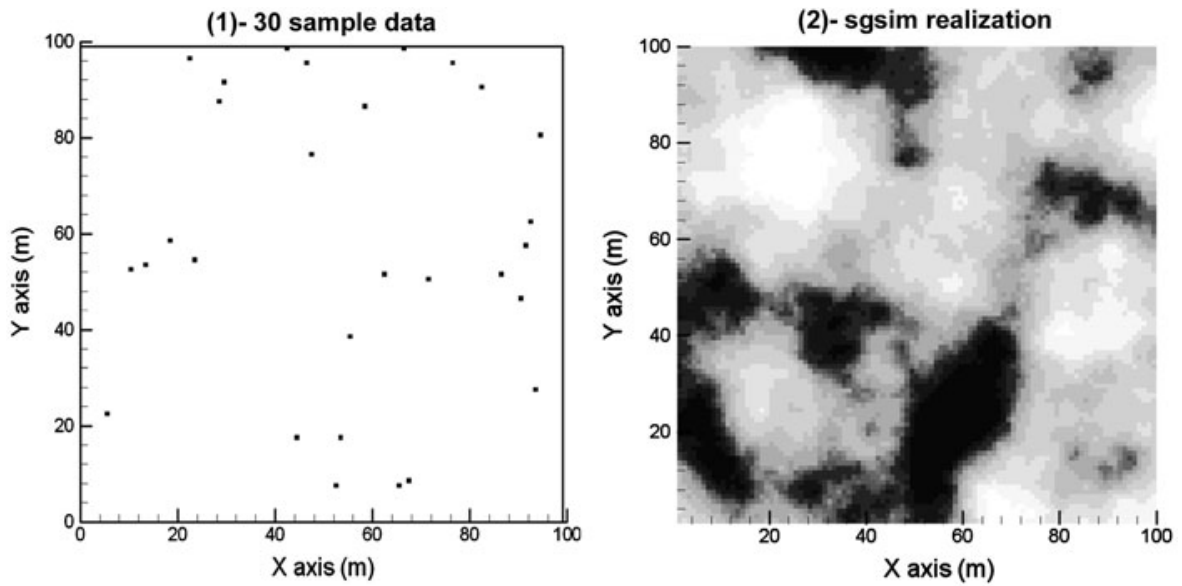

(3)- filtersim realization

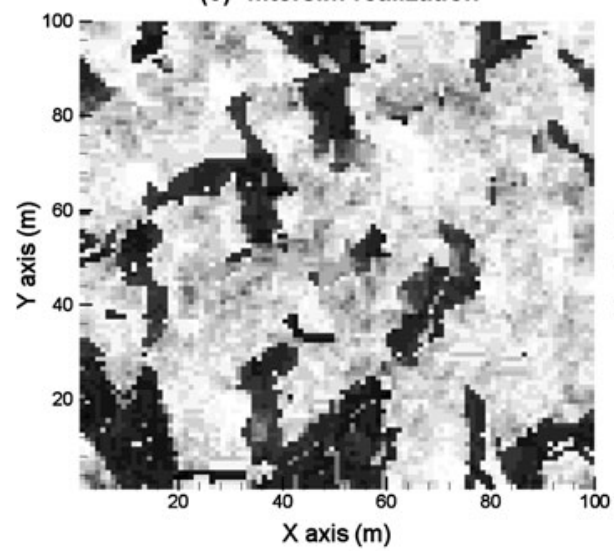

(4)- hosim realization

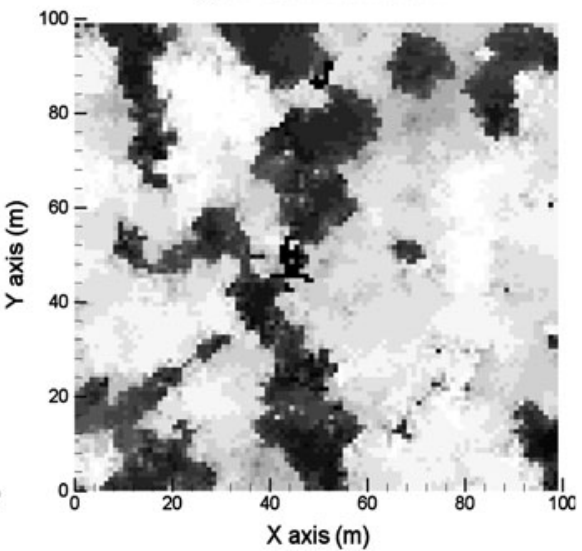

Fig. 26 Simulation of the 2D section presented in Fig. 9(1). (1) 30 sample data, (2) one sgsim realization, (3) one filtersim realization, and (4) one hosim realization

realizations generated by hosim are very close to the exhaustive image using either DS-2 (85 samples) or any of the above training images. However, spatial connectivity in the exhaustive image may not be well captured when using DS-3; in this case, the estimated cumulants and, then, the generated realizations would be more related to the TI used.

\subsection{Example 5: A Comparison of hosim, sgsim, and fitersim}

The numerical examples presented above aimed to present the main features of the proposed method. In this last example, we provide a small comparison between hosim, sequential Gaussian simulation (sgsim), and filtersim algorithms. Different realizations are generated by the different algorithms to simulate the exhaustive image using 30 sample data, as shown in Fig. 26(1); TI-1 is used by hosim and filtersim. An ellipsoid of size $(20,20)$, a spherical variogram of range 40, sill 1, and nugget 
0 are used to calculate the covariance by sgsim; a template with size $15 \times 15$ and 5 multigrids are used by filtersim. Figures 26(2) to (4) present the realizations generated by sgsim, filtersim, and hosim, respectively. This figure shows the superiority of filtersim and hosim over sgsim in producing continuity of the NS channels. The effects of data are evidently larger in the realizations generated with hosim than with filtersim; firstly, the couple of data in the range [60-80] along $x$ and [0-60] $y$ clear that area from the TI-1 to reproduce the corresponding area in the exhaustive image; in addition, about two large channels are very well reproduced and characterize the direction of the anisotropy presented in the exhaustive image. Lastly, the information in the range [80-100] along $x$ and [0-100] $y$ better describe the small channels seen in the exhaustive image.

\section{Conclusions}

This paper presented a new high-order sequential simulation method using the concept of high-order spatial cumulants. The sequential algorithm developed is based on a high-order approximation of the local probability density function using continuous multivariate Legendre series, with coefficients calculated from spatial cumulants. The approach developed uses different orders of cumulants, contrary to the present MP-based algorithms. The algorithm presented herein is tested in simulating a 2D horizontal section from a channel complex using 361 samples. Different realizations are generated showing an excellent reproduction of data statistics and high-order statistics, that is, the histogram, experimental variograms, and high-order spatial statistics of the sample data. The concept of TI is used in a second example with different sparser sample data. In this case, realizations which honor the data and their statistics are generated as well. Unconditionally simulated realizations are generated to better demonstrate the effects of the available data. An additional example assesses the sensitivity of the method developed to the TIs used. The results show that the newly proposed method is much less sensitive to the TIs than the existing MP methods due to the data-driven approach of the method. Finally, a comparison between sgsim and filtersim shows a better performance of the algorithm developed. In future work, we will consider the extension of the method to the simulation directly at block support, the development of a cumulant-based method to simulate categorical data, and improve the efficiency of the method.

Acknowledgements The work in this paper was funded from NSERC CDR Grant 335696 and BHP Billiton, and from NSERC Discovery Grant 239019. Thanks are in order to Brian Baird, Peter Stone, and Gavin Yates of BHP Billiton, to BHP Billiton Diamonds, and, in particular, Darren Dyck, for their support, collaboration, and technical comments.

Open Access This article is distributed under the terms of the Creative Commons Attribution Noncommercial License which permits any noncommercial use, distribution, and reproduction in any medium, provided the original author(s) and source are credited.

\section{Appendix A: Calculation of High-order Spatial Cumulants}

The translation of high-order moments to high-order cumulants, and vice versa, can be obtained recursively (Dimitrakopoulos et al. 2010; Mustapha and Dimitrakopoulos 
2010) as

$$
\begin{aligned}
E\left(Z_{1}^{i_{1}} \cdots Z_{n}^{i_{n}}\right)= & m_{i_{1} \ldots i_{n}}=\sum_{j_{1}=0}^{i_{1}} \cdots \sum_{j_{1}=0}^{i_{n-1}} \sum_{j_{1}=0}^{i_{n}-1}\left(\begin{array}{c}
i_{1} \\
j_{1}
\end{array}\right) \ldots\left(\begin{array}{c}
i_{n-1} \\
j_{n-1}
\end{array}\right)\left(\begin{array}{c}
i_{n}-1 \\
j_{n}
\end{array}\right) \\
& \times c_{i_{1}-j_{1}, \ldots, i_{n-1}-j_{n-1}, i_{n}-j_{n}} m_{j_{1}, \ldots, j_{n-1}, j_{n}}
\end{aligned}
$$

and

$$
\begin{gathered}
\operatorname{Cum}\left(Z_{1}^{i_{1}} \cdots Z_{n}^{i_{n}}\right)=c_{i_{1} \ldots i_{n}}=\sum_{j_{1}=0}^{i_{1}} \cdots \sum_{j_{1}=0}^{i_{n-1}} \sum_{j_{1}=0}^{i_{n}-1}\left(\begin{array}{c}
i_{1} \\
j_{1}
\end{array}\right) \ldots\left(\begin{array}{c}
i_{n-1} \\
j_{n-1}
\end{array}\right)\left(\begin{array}{c}
i_{n}-1 \\
j_{n}
\end{array}\right) \\
\times m_{i_{1}-j_{1}, \ldots, i_{n-1}-j_{n-1}, i_{n}-j_{n}} c_{j_{1}, \ldots, j_{n-1}, j_{n}} .
\end{gathered}
$$

Assuming that $\mathbf{Z}(\mathbf{x})$ is a zero-mean ergodic stationary random field indexed in $R^{n}$, then the $r$ th-order moment of the random field is defined as $E(\mathbf{Z}(\mathbf{x}) \mathbf{Z}(\mathbf{x}+$ $\left.\left.h_{1}\right) \cdots \mathbf{Z}\left(\mathbf{x}+h_{r-1}\right)\right)$. The moments depend only on $h_{1}, \ldots, h_{r-1}$. Similarly, the $r$ thorder cumulant can be denoted as $c_{i_{1}, \ldots, i_{n}}\left(h_{1}, \ldots, h_{r-1}\right)$, where $r=i_{1}+\cdots+i_{n}$. For example, the second-order cumulants of a noncentered random function $\mathbf{Z}(\mathbf{x})$, known as the covariance, is given using (A.2) by

$$
c_{1,1}(h)=E\left(\mathbf{Z}(\mathbf{x}) \mathbf{Z}\left(\mathbf{x}+h_{1}\right)\right)-E(\mathbf{Z}(\mathbf{x}))^{2} .
$$

Its third-order cumulant is given by

$$
\begin{aligned}
c_{1,1,1}\left(h_{1}, h_{2}\right)= & E\left(\mathbf{Z}(\mathbf{x}) \mathbf{Z}\left(\mathbf{x}+h_{1}\right) \mathbf{Z}\left(\mathbf{x}+h_{2}\right)\right) \\
& -E(\mathbf{Z}(\mathbf{x})) E\left(\mathbf{Z}\left(\mathbf{x}+h_{1}\right) \mathbf{Z}\left(\mathbf{x}+h_{2}\right)\right) \\
& -E(\mathbf{Z}(\mathbf{x})) E\left(\mathbf{Z}\left(\mathbf{x}+h_{1}\right) \mathbf{Z}\left(\mathbf{x}+h_{3}\right)\right) \\
& -E(\mathbf{Z}(\mathbf{x})) E\left(\mathbf{Z}\left(\mathbf{x}+h_{2}\right) \mathbf{Z}\left(\mathbf{x}+h_{3}\right)\right)+2 E(\mathbf{Z}(\mathbf{x}))^{3},
\end{aligned}
$$

where $h_{3}$ is along the difference between the vectors supporting $h_{1}$ and $h_{2}$. The cumulants are invariant to additive constants; thus, if a given process $\mathbf{Z}(\mathbf{x})$ is not zeromean, its cumulants can be computed as the cumulants of $\mathbf{Z}(\mathbf{x})-E(\mathbf{Z}(\mathbf{x}))$ (Nikias and Petropulu 1993). It can be computationally convenient to consider zero-mean random functions as some of the terms vanish. Then, the following expression is used by Mustapha and Dimitrakopoulos (2010) to calculate the $\left(j_{0}+j_{1}+j_{2}+j_{3}\right)$-th-order cumulant

$$
\begin{aligned}
\operatorname{cum} & \left(Z_{0}^{j_{0}}, Z_{1}^{j_{1}}, Z_{2}^{j_{2}}, Z_{3}^{j_{3}}\right) \\
= & \frac{1}{N} \sum_{k=1}^{N} \mathbf{Z}\left(x_{k}\right)^{j_{0}} \mathbf{Z}\left(x_{k}+h_{1}\right)^{j_{1}} \mathbf{Z}\left(x_{k}+h_{2}\right)^{j_{2}} \mathbf{Z}\left(x_{k}+h_{3}\right)^{j_{3}} \mathbf{Z}\left(x_{k}+h_{4}\right)^{j_{4}} \\
& -\frac{1}{N^{2}}\left[\left(\sum_{k=1}^{N} \mathbf{Z}\left(x_{k}\right)^{j_{0}} \mathbf{Z}\left(x_{k}+h_{1}\right)^{j_{1}}\right)\right.
\end{aligned}
$$


$\left.\times\left(\sum_{k=1}^{N} \mathbf{Z}\left(x_{k}+h_{2}\right)^{j_{2}} \mathbf{Z}\left(x_{k}+h_{3}\right)^{j_{3}} \mathbf{Z}\left(x_{k}+h_{4}\right)^{j_{4}}\right)\right]$

$-\frac{1}{N^{2}}\left[\left(\sum_{k=1}^{N} \mathbf{Z}\left(x_{k}\right)^{j_{0}} \mathbf{Z}\left(x_{k}+h_{2}\right)^{j_{2}}\right)\right.$

$\left.\times\left(\sum_{k=1}^{N} \mathbf{Z}\left(x_{k}+h_{1}\right)^{j_{1}} \mathbf{Z}\left(x_{k}+h_{3}\right)^{j_{3}} \mathbf{Z}\left(x_{k}+h_{4}\right)^{j_{4}}\right)\right]$

$-\frac{1}{N^{2}}\left[\left(\sum_{k=1}^{N} \mathbf{Z}\left(x_{k}\right)^{j_{0}} \mathbf{Z}\left(x_{k}+h_{3}\right)^{j_{3}}\right)\right.$

$\left.\times\left(\sum_{k=1}^{N} \mathbf{Z}\left(x_{k}+h_{1}\right)^{j_{1}} \mathbf{Z}\left(x_{k}+h_{2}\right)^{j_{2}} \mathbf{Z}\left(x_{k}+h_{4}\right)^{j_{4}}\right)\right]$

$-\frac{1}{N^{2}}\left[\left(\sum_{k=1}^{N} \mathbf{Z}\left(x_{k}\right)^{j_{0}} \mathbf{Z}\left(x_{k}+h_{4}\right)^{j_{4}}\right)\right.$

$\left.\times\left(\sum_{k=1}^{N} \mathbf{Z}\left(x_{k}+h_{1}\right)^{j_{1}} \mathbf{Z}\left(x_{k}+h_{2}\right)^{j_{2}} \mathbf{Z}\left(x_{k}+h_{3}\right)^{j_{3}}\right)\right]$

$-\frac{1}{N^{2}}\left[\left(\sum_{k=1}^{N} \mathbf{Z}\left(x_{k}+h_{1}\right)^{j_{1}} \mathbf{Z}\left(x_{k}+h_{2}\right)^{j_{2}}\right)\right.$

$\left.\times\left(\sum_{k=1}^{N} \mathbf{Z}\left(x_{k}\right)^{j_{0}} \mathbf{Z}\left(x_{k}+h_{3}\right)^{j_{3}} \mathbf{Z}\left(x_{k}+h_{4}\right)^{j_{4}}\right)\right]$

$-\frac{1}{N^{2}}\left[\left(\sum_{k=1}^{N} \mathbf{Z}\left(x_{k}+h_{1}\right)^{j_{1}} \mathbf{Z}\left(x_{k}+h_{3}\right)^{j_{3}}\right)\right.$

$\left.\times\left(\sum_{k=1}^{N} \mathbf{Z}\left(x_{k}\right)^{j_{0}} \mathbf{Z}\left(x_{k}+h_{2}\right)^{j_{2}} \mathbf{Z}\left(x_{k}+h_{4}\right)^{j_{4}}\right)\right]$

$-\frac{1}{N^{2}}\left[\left(\sum_{k=1}^{N} \mathbf{Z}\left(x_{k}+h_{1}\right)^{j_{1}} \mathbf{Z}\left(x_{k}+h_{4}\right)^{j_{4}}\right)\right.$

$\left.\times\left(\sum_{k=1}^{N} \mathbf{Z}\left(x_{k}\right)^{j_{0}} \mathbf{Z}\left(x_{k}+h_{2}\right)^{j_{2}} \mathbf{Z}\left(x_{k}+h_{3}\right)^{j_{3}}\right)\right]$

$-\frac{1}{N^{2}}\left[\left(\sum_{k=1}^{N} \mathbf{Z}\left(x_{k}+h_{2}\right)^{j_{2}} \mathbf{Z}\left(x_{k}+h_{3}\right)^{j_{3}}\right)\right.$ 


$$
\begin{aligned}
& \left.\times\left(\sum_{k=1}^{N} \mathbf{Z}\left(x_{k}\right)^{j_{0}} \mathbf{Z}\left(x_{k}+h_{1}\right)^{j_{1}} \mathbf{Z}\left(x_{k}+h_{4}\right)^{j_{4}}\right)\right] \\
& -\frac{1}{N^{2}}\left[\left(\sum_{k=1}^{N} \mathbf{X}\left(x_{k}+h_{2}\right)^{j_{2}} \mathbf{X}\left(x_{k}+h_{4}\right)^{j_{4}}\right)\right. \\
& \left.\times\left(\sum_{k=1}^{N} \mathbf{X}\left(x_{k}\right)^{j_{0}} \mathbf{X}\left(x_{k}+h_{1}\right)^{j_{1}} \mathbf{X}\left(x_{k}+h_{3}\right)^{j_{3}}\right)\right] \\
& -\frac{1}{N^{2}}\left[\left(\sum_{k=1}^{N} \mathbf{X}\left(x_{k}+h_{3}\right)^{j_{3}} \mathbf{X}\left(x_{k}+h_{4}\right)^{j_{4}}\right)\right. \\
& \left.\times\left(\sum_{k=1}^{N} \mathbf{X}\left(x_{k}\right)^{j_{0}} \mathbf{X}\left(x_{k}+h_{1}\right)^{j_{1}} \mathbf{X}\left(x_{k}+h_{2}\right)^{j_{2}}\right)\right],
\end{aligned}
$$

where $N$ is the number of elements in the set $T^{h_{1}, h_{2}, h_{3}, h_{4}}$ defined by (17).

\section{Appendix B: Formula for Legendre Coefficients}

In this appendix, we derive an expression of Legendre series coefficients in terms of cumulants. The method is shown in $1 \mathrm{D}$ and can be easily generalized. Consider the coefficient of order $m$ in (10). The coefficient $L_{m}$, given by

$$
L_{m}=\int_{D} \bar{P}_{m}(z) f(z) d z,
$$

can be written using (5) as

$$
L_{m}=\sqrt{\frac{2 m+1}{2}} \int_{D} \sum_{i=0}^{m} a_{i, m} z^{i} f(z) d z
$$

Then, by permuting the integral and the sum in (B.2), we get

$$
L_{m}=\sqrt{\frac{2 m+1}{2}} \sum_{i=0}^{m} a_{i, m} \int_{D} z^{i} f(z) d z .
$$

Using (A.2), (B.3) can be expressed as

$$
\begin{aligned}
L_{m} & =\sqrt{\frac{2 m+1}{2}} \sum_{i=0}^{m} \sum_{j=0}^{i}\left(\begin{array}{l}
i \\
j
\end{array}\right) a_{i, m} c_{i-j} m_{j}=g_{m}\left(c_{i}\right), \\
i & =0, \ldots, m \text { and } m=0,1,2, \ldots
\end{aligned}
$$




\section{References}

Alabert FG (1987) Stochastic imaging of spatial distributions using hard and soft information. MSc thesis, Stanford University

Arpat B, Caers J (2007) Stochastic simulation with patterns. Math Geosci 39:177-203

Bernardeau F, Colombi S, Gaztanaga E, Scoccimarro R (2002) Large-scale structure of the universe and cosmological perturbation theory. Phys Rep 367(1):1-248

Billinger DR, Rosenblatt M (1966) Asymptotic theory of $k$ th-order spectra. In: Harris B (ed) Spectral analysis of time series. Wiley, New York, pp 189-232

Boucher A (2009) Considering complex training images with search tree partitioning. Comput Geosci 35:1151-1158

Chatterjee S, Dimitrakopoulos R, Mustapha H (2009) Three-dimensional wavelet based conditional cosimulation using training image. COSMO Res Rep 3(2):143-181

Chilès JP, Delfiner P (1999) Geostatistics—modeling spatial uncertainty. Wiley, New York

Chugunova TL, Hu LY (2008) Multiple point simulations constrained by continuous auxiliary data. Math Geosci 40:133-146

Daly C (2004) Higher order models using entropy, Markov random fields and sequential simulation. In: Geostatistics Banff. Springer, Berlin, pp 215-225

Daniels HE (1954) Saddlepoint approximations in statistics. Ann Math Stat 25:631-650

David M (1988) Handbook of applied advanced geostatistical ore reserve estimation. Elsevier, Amsterdam

Delopoulos A, Giannakis GB (1996) Cumulant based identification of noisy closed loop systems. Int J Adapt Control Signal Process 10(2/3):303-317

Dimitrakopoulos R, Mustapha H, Gloaguen E (2010) High-order statistics of spatial random fields: exploring spatial cumulants for modelling complex, non-Gaussian and non-linear phenomena. Math Geosci 42:65-99

Dimitrakopoulos R (1990) Conditional simulation of intrinsic random functions of order $k$. Math Geosci 22:361-380

Dimitrakopoulos R, Luo X (2004) Generalized sequential Gaussian simulation. Math Geosci 36:567-591

Edgeworth FY (1905) The law of error. Trans Camb Philos Soc 20:113-141

Edgeworth FY (1907) On the representation of a statistical frequency by a series. J R Stat Soc 70:102-106

Gaztanaga EP, Fosalba P, Elizalde E (2000) Gravitational evolution of the large-scale probability density distribution. Astrophys J 539:522-531

Goovaerts P (1998) Geostatistics for natural resources evaluation. Oxford, New York

Gloaguen E, Dimitrakopoulos R (2009) Two-dimensional conditional simulations based on the wavelet decomposition of training images. Math Geol 41:679-701

Guardiano J, Srivastava RM (1993) Multivariate geostatistics: beyond bivariate moments. In: Soares A (ed) Geosatistics Tróia '92, vol 1. Kluwer, Dordrecht, pp 133-144

Hille E (1926) On Laguerre's Series: First note. Proc Natl Acad Sci USA 12:261-265

Hosny KM (2007) Exact Legendre moment computation for gray level images. Pattern Recogn 40:35973605

Journel AG (1994) Modelling uncertainty: some conceptual thoughts. In: Dimitrakopoulos R (ed) Geostatistics for the next century. Kluwer Academic Press, Dordrecht, pp 30-43

Journel AG (1997) Deterministic geostatistics: a new visit. In: Baafy E, Shofield N (eds) Geostatistics Woolongong '96. Kluwer, Dordrecht, pp 213-224

Journel AG, Alabert F (1989) Non-Gaussian data expansion in the earth sciences. Terra Nova 1:123-134

Kendall MG, Stuart A (1977) The advanced theory of statistics, 4th edn, vol 1. Macmillan, New York

Law AM, Kelton WD (1999) Simulation modelling and analysis. McGraw-Hill Higher Education, Singhapore

Lebedev NN (1965) Special functions and their applications. Prentice-Hall Inc., New York

Liao SX, Pawlak M (1996) On image analysis by moments. IEEE Trans Pattern Anal Mach Intell 18:254266

Mao S, Journel AG (1999) Generation of a reference petrophysical and seismic 3D data set: the Stanford V reservoir. In: Stanford Center for Reservoir Forecasting Annual Meeting. Available at: http://ekofisk. stanford.edu/SCRF.html

Mirowski PW, Trtzlaff DM, Davies RC, McCormick DS, Williams N, Signer C (2008) Stationary scores on training images for multipoint geostatistics. Math Geosci 41:447-474

Mustapha H, Dimitrakopoulos R (2010) A new approach for geological pattern recognition using highorder spatial cumulants. Comput Geosci 36:313-343 
Nikias CL, Petropulu AP (1993) Higher-order spectra analysis: a nonlinear signal processing framework. PTR Prentice Hall, Upper Saddle River

Pan J Szapudi I (2005) Conditional cumulants in a weakly non-linear regime. Mon Not R Astron Soc $361: 357-361$

Remy N, Boucher A, Wu J (2009) Applied geostatistics with SGeMs: a users's guide. Cambridge University Press, Cambridge

Ripley BD (1987) Stochastic simulation. John Wiley \& Sons, New York

Rosenblatt M (1985) Stationary sequences and random fields. Birkhaüser, Boston

Rubinstein R (1981) Simulation and the Monte Carlo method. John Wiley \& Sons, New York

Sadler B, Giannakis GB, Shamsunder S (1995) Noise subspace techniques in non-Gaussian noise using cumulants. IEEE Trans Aerosp Electron Syst 31:1009-1018

Scheidt C, Caers J (2009) Representing spatial uncertainty using distances and kernels. Math Geosci 41:397-419

Spiegel MR (1968) Mathematical handbook of formulas and tables. Schaum's outline series. McGraw-Hill Book Co., New York

Strebelle S (2002) Conditional simulation of complex geological structures using multiple point statistics. Math Geosci 34:1-22

Tjelmeland H (1998) Markov random fields with higher order interactions. Scand J Stat 25:415-433

Tjelmeland H, Eidsvik J (2004) Directional metropolis: hastings updates for conditionals with nonlinear likelihoods. In: Geostatistics Banff, vol 1. Springer, Berlin, pp 95-104

Volker O, Dimitrakopoulos R (2007) Simulation of wireframes and geometric features with multiple point techniques: application at Yandi Iron Ore deposit, Australia. Orebody modelling and strategic mine planning, The Australasian Institute of Mining and Metallurgy, Spectrum Series, vol 14, pp 51-60

Welling M (1999) Robust series expansions for probability density estimation. Technical report, California Institute of Technology, Computational Vision Lab

Wilson GA, Wragg A (1973) Numerical methods for approximating continuous probability density functions over [0,) using moments. IMA J Appl Math 12:165-173

Wu J, Boucher A, Zhang T (2008) SGeMS code for pattern simulation of continuous and categorical variables: FILTERSIM. Comput Geosci 34:1863-1876

Yap PT, Paramesran R (2005) An efficient method for the computation of Legendre moments. IEEE Trans Pattern Anal Mach Intell 27:1996-2002

Zhang F (2005) A high order cumulants based multivariate nonlinear blind source separation method source. Mach Learn J 61105-61127

Zhang T, Switzer P, Journel AG (2006) Filter-based classification of training image patterns for spatial simulation. Math Geol 38:63-80 